\title{
Robust Understanding in Multimodal Interfaces
}

\author{
Srinivas Bangalore* \\ AT\&T Labs - Research \\ Michael Johnston** \\ AT\&T Labs - Research
}

Multimodal grammars provide an effective mechanism for quickly creating integration and understanding capabilities for interactive systems supporting simultaneous use of multiple input modalities. However, like other approaches based on hand-crafted grammars, multimodal grammars can be brittle with respect to unexpected, erroneous, or disfluent input. In this article, we show how the finite-state approach to multimodal language processing can be extended to support multimodal applications combining speech with complex freehand pen input, and evaluate the approach in the context of a multimodal conversational system (MATCH). We explore a range of different techniques for improving the robustness of multimodal integration and understanding. These include techniques for building effective language models for speech recognition when little or no multimodal training data is available, and techniques for robust multimodal understanding that draw on classification, machine translation, and sequence edit methods. We also explore the use of edit-based methods to overcome mismatches between the gesture stream and the speech stream.

\section{Introduction}

The ongoing convergence of the Web with telephony, driven by technologies such as voice over IP, broadband Internet access, high-speed mobile data networks, and handheld computers and smartphones, enables widespread deployment of multimodal interfaces which combine graphical user interfaces with natural modalities such as speech and pen. The critical advantage of multimodal interfaces is that they allow user input and system output to be expressed in the mode or modes to which they are best suited, given the task at hand, user preferences, and the physical and social environment of the interaction (Oviatt 1997; Cassell 2001; André 2002; Wahlster 2002). There is also an increasing body of empirical evidence (Hauptmann 1989; Nishimoto et al. 1995; Cohen et al. 1998a; Oviatt 1999) showing user preference and task performance advantages of multimodal interfaces.

In order to support effective multimodal interfaces, natural language processing techniques, which have typically operated over linear sequences of speech or text,

\footnotetext{
* 180 Park Avenue, Florham Park, NJ 07932. E-mail: srini@research.att.com.

** 180 Park Avenue, Florham Park, NJ 07932. E-mail: johnston@research.att.com.
}

Submission received: 26 May 2006; revised submission received: 6 May 2008; accepted for publication: 11 July 2008. 
need to be extended in order to support integration and understanding of multimodal language distributed over multiple different input modes (Johnston et al. 1997; Johnston 1998b). Multimodal grammars provide an expressive mechanism for quickly creating language processing capabilities for multimodal interfaces supporting input modes such as speech and gesture (Johnston and Bangalore 2000). They support composite multimodal inputs by aligning speech input (words) and gesture input (represented as sequences of gesture symbols) while expressing the relation between the speech and gesture input and their combined semantic representation. Johnston and Bangalore (2005) show that such grammars can be compiled into finite-state transducers, enabling effective processing of lattice input from speech and gesture recognition and mutual compensation for errors and ambiguities.

In this article, we show how multimodal grammars and their finite-state implementation can be extended to support more complex multimodal applications. These applications combine speech with complex pen input including both freehand gestures and handwritten input. More general mechanisms are introduced for representation of gestures and abstraction over specific content in the gesture stream along with a new technique for aggregation of gestures. We evaluate the approach in the context of the MATCH multimodal conversational system (Johnston et al. 2002b), an interactive city guide. In Section 2, we present the MATCH application, the architecture of the system, and our experimental method for collection and annotation of multimodal data. In Section 3, we evaluate the baseline approach on the collected data.

The performance of this baseline approach is limited by the use of hand-crafted models for speech recognition and multimodal understanding. Like other approaches based on hand-crafted grammars, multimodal grammars can be brittle with respect to extra-grammatical, erroneous, and disfluent input. This is particularly problematic for multimodal interfaces if they are to be used in noisy mobile environments. To overcome this limitation we explore a broad range of different techniques for improving the robustness of both speech recognition and multimodal understanding components.

For automatic speech recognition (ASR), a corpus-driven stochastic language model (SLM) with smoothing can be built in order to overcome the brittleness of a grammarbased language model. However, for multimodal applications there is often very little training data available and collection and annotation of realistic data can be very expensive. In Section 5, we examine and evaluate various different techniques for rapid prototyping of the language model for the speech recognizer, including transformation of out-of-domain data, grammar sampling, adaptation from wide-coverage grammars, and speech recognition models built on conversational corpora (Switchboard). Although some of the techniques presented have been reported in the literature, we are not aware of work comparing the effectiveness of these techniques on the same domain and using the same data sets. Furthermore, the techniques are general enough that they can be applied to bootstrap robust gesture recognition models as well. The presentation here focuses on speech recognition models, partly due to the greater impact of speech recognition performance compared to gesture recognition performance on the multimodal application described here. However, in Section 7 we explore the use of robustness techniques on gesture input.

Although the use of an SLM enables recognition of out-of-grammar utterances, resulting in improved speech recognition accuracy, this may not help overall system performance unless the multimodal understanding component itself is made robust to unexpected inputs. In Section 6, we describe and evaluate several different techniques for making multimodal understanding more robust. Given the success of discriminative classification models in related applications such as natural language call 
routing (Haffner, Tur, and Wright 2003; Gupta et al. 2004) and semantic role labeling (Punyakanok, Roth, and Yih 2005), we first pursue a purely data-driven approach where the predicate of a multimodal command and its arguments are determined by classifiers trained on an annotated corpus of multimodal data. However, given the limited amount of data available, this approach does not provide an improvement over the grammar-based approach. We next pursue an approach combining grammar and data where robust understanding is viewed as a statistical machine translation problem where out-of-grammar or misrecognized language must be translated to the closest language the system can understand. This approach provides modest improvement over the grammar-based approach. Finally we explore an edit-distance approach which combines grammar-based understanding with knowledge derived from the underlying application database. Essentially, if a string cannot be parsed, we attempt to identify the in-grammar string that it is most similar to, just as in the translation approach. This is achieved by using a finite-state edit transducer to compose the output of the ASR with the grammar-based multimodal alignment and understanding models. We have presented these techniques as methods for improving the robustness of the multimodal understanding by processing the speech recognition output. Given the higher chance of error in speech recognition compared to gesture recognition, we focus on processing the speech recognition output to achieve robust multimodal understanding. However, these techniques are also equally applicable to gesture recognition output. In Section 7, we explore the use of edit techniques on gesture input. Section 8 concludes and discusses the implications of these results.

\section{The MATCH Application}

Urban environments present a complex and constantly changing body of information regarding restaurants, cinema and theater schedules, transportation topology, and timetables. This information is most valuable if it can be delivered effectively while mobile, since users' needs change rapidly and the information itself is dynamic (e.g., train times change and shows get cancelled). MATCH (Multimodal Access To City Help) is a working city guide and navigation system that enables mobile users to access restaurant and subway information for urban centers such as New York City and Washington, DC (Johnston et al. 2002a, 2002b). MATCH runs stand-alone on a tablet PC (Figure 1) or in client-server mode across a wireless network. There is also a kiosk version of the system (MATCHkiosk) (Johnston and Bangalore 2004) which incorporates a life-like talking head. In this article, we focus on the mobile version of $\mathrm{MATCH}$, in which the user interacts with a graphical interface displaying restaurant listings and a dynamic map showing locations and street information. The inputs can be speech, drawings on the display with a stylus, or synchronous multimodal combinations of the two modes. The user can ask for reviews, cuisine, phone number, address, or other information about restaurants and for subway directions to restaurants and locations. The system responds with graphical callouts on the display, synchronized with synthetic speech output.

For example, a user can request to see restaurants using the spoken command show cheap italian restaurants in chelsea. The system will then zoom to the appropriate map location and show the locations of restaurants on the map. Alternatively, the user could give the same command multimodally by circling an area on the map and saying show cheap italian restaurants in this neighborhood. If the immediate environment is too noisy or public, the same command can be given completely using a pen stylus as in Figure 2, by circling an area and writing cheap and italian. 


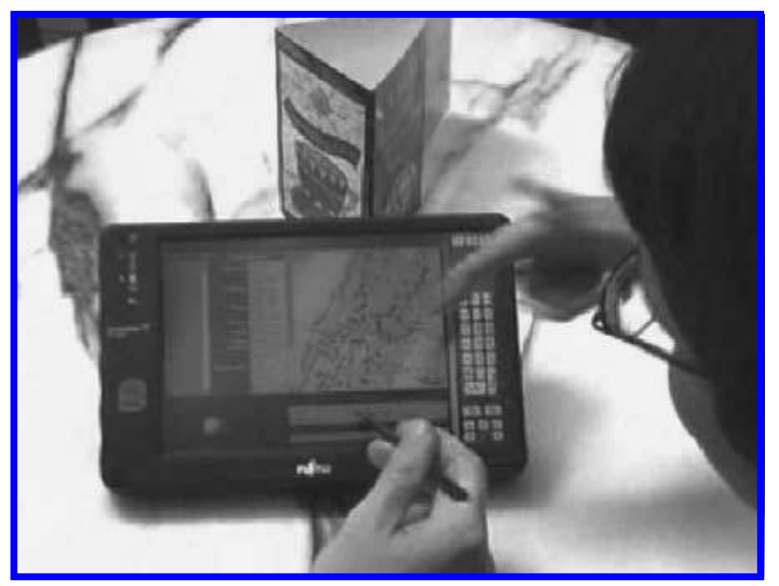

Figure 1

MATCH on tablet.

Similarly, if the user says phone numbers for these two restaurants and circles two restaurants as in Figure 3(a) [A], the system will draw a callout with the restaurant name and number and say, for example, Time Cafe can be reached at 212-533-7000, for each restaurant in turn (Figure 3(a) [B]). If the immediate environment is too noisy or public, the same command can be given completely in pen by circling the restaurants and writing phone (Figure 3(b)).

The system also provides subway directions. For example, if the user says How do I get to this place? and circles one of the restaurants displayed on the map the system will ask Where do you want to go from?. The user can then respond with speech (for example, 25th Street and 3rd Avenue), with pen by writing (for example, 25th St E 3rd Ave), or multimodally (for example, from here, with a circle gesture indicating the location). The system then calculates the optimal subway route and generates a multimodal presentation coordinating graphical presentation of each stage of the route with spoken instructions indicating the series of actions the user needs to take (Figure 4).

Map-based systems have been a common application area for exploring multimodal interaction techniques. One of the reasons for this is the effectiveness and naturalness of combining graphical input to indicate spatial locations with spoken input to specify commands. See Oviatt (1997) for a detailed experimental investigation illustrating the

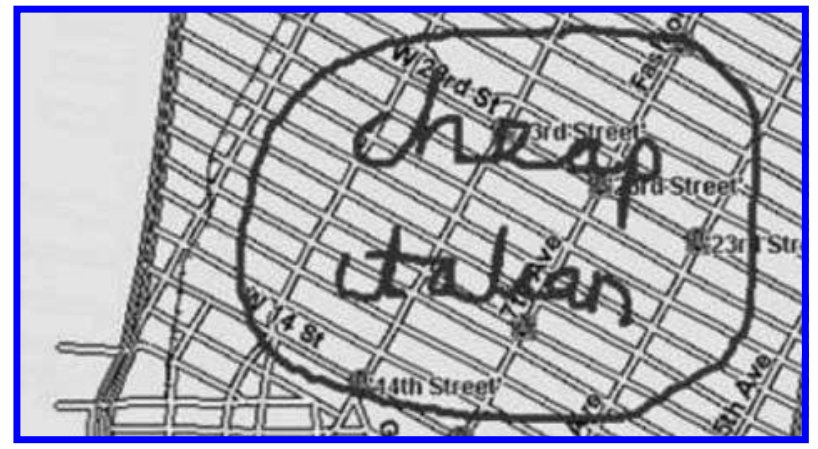

Figure 2

Unimodal pen command. 


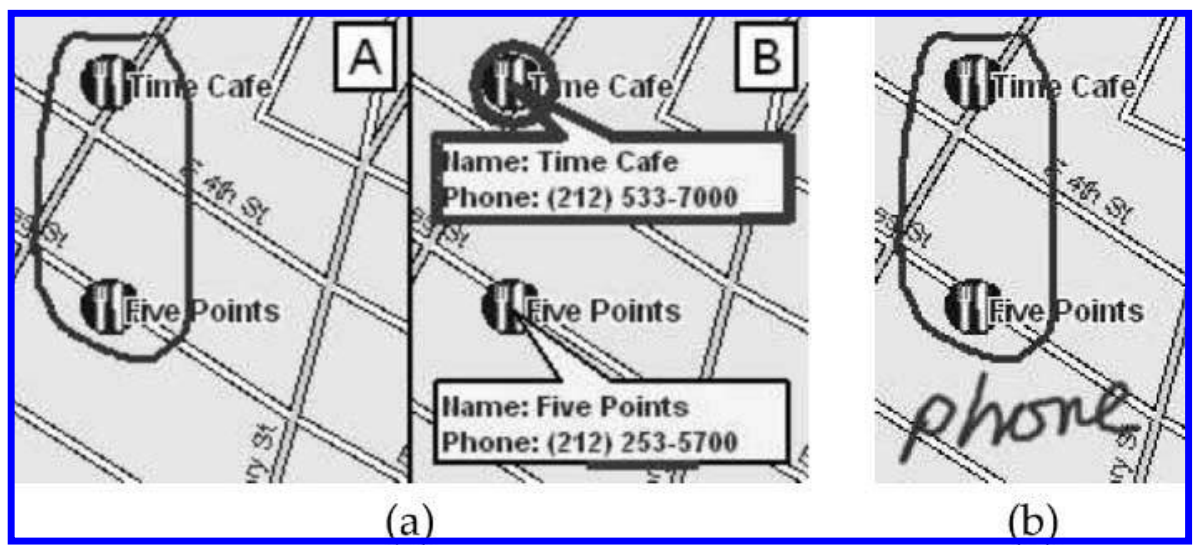

Figure 3

(a) Two area gestures. (b) Phone command in pen.

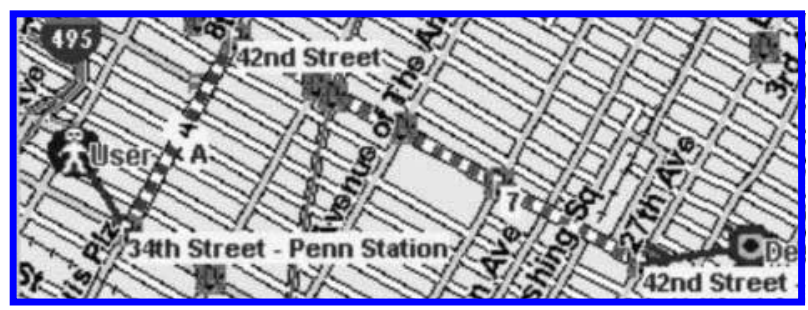

Figure 4

Multimodal subway route.

advantages of multimodal input for map-based tasks. Previous map-based multimodal prototypes can be broken down into two main task domains: map annotation tasks and information search tasks. Systems such as QuickSet (Cohen et al. 1998b) focus on the use of speech and pen input in order to annotate the location of features on a map. Other systems use speech and pen input to enable users to search and browse for information through direct interaction with a map display. In the ADAPT system (Gustafson et al. 2000), users browse for apartments using combinations of speaking and pointing. In the Multimodal Maps system (Cheyer and Julia 1998), users perform travel planning tasks such as searching for hotels and points of interest. MATCH is an information search application providing local search capabilities combined with transportation directions. As such it is most similar to the Multimodal Maps application, though it provides more powerful and robust language processing and multimodal integration capabilities, while the language processing in the Multimodal Maps application is limited to simple Verb Object Argument constructions (Cheyer and Julia 1998).

In the next section we explain the underlying architecture and the series of components which enable the MATCH user interface.

\subsection{MATCH Multimodal Architecture}

The underlying architecture that supports MATCH consists of a series of re-usable components which communicate over IP through a facilitator (MCUBE) (Figure 5). Figure 6 shows the flow of information among components in the system. In earlier 


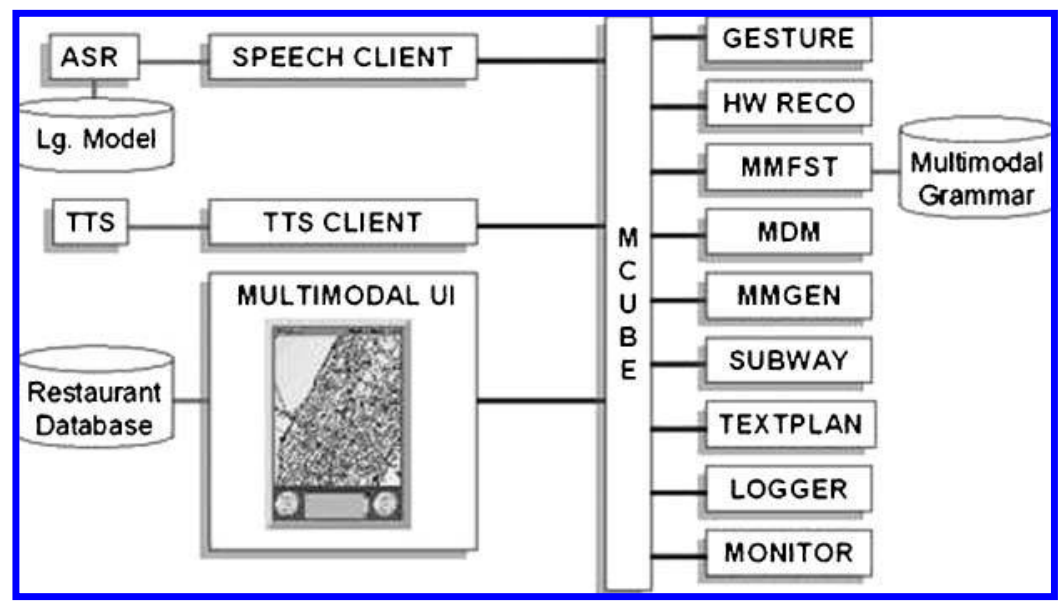

Figure 5

Multimodal architecture.

versions of the system, communication was over socket connections. In later versions of the system communication between components uses HTTP.

Users interact with the system through a Multimodal User Interface client (MUI) which runs in a Web browser. Their speech is processed by the WATSON speech recognition server (Goffin et al. 2005) resulting in a weighted lattice of word strings. When the user draws on the map their ink is captured and any objects potentially selected, such as currently displayed restaurants, are identified. The electronic ink is broken into a lattice of strokes and sent to both gesture and handwriting recognition components which

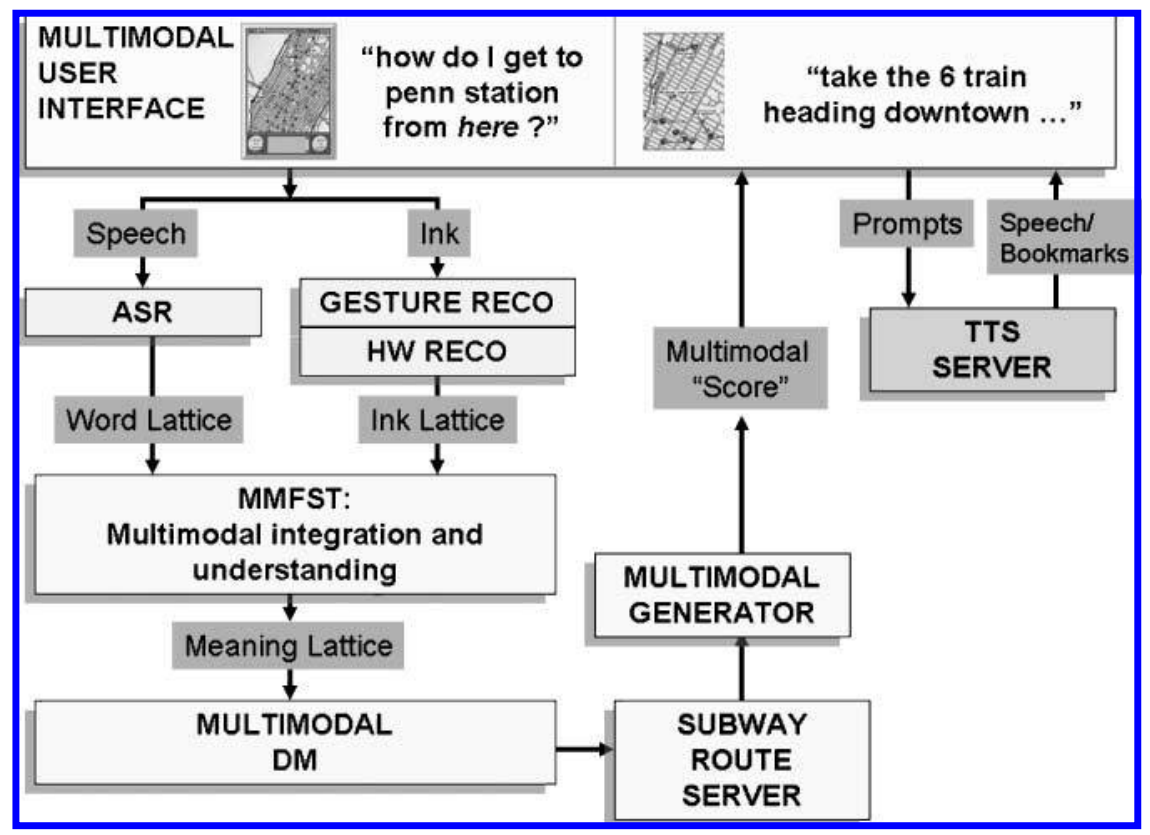

Figure 6

Multimodal architecture flowchart. 
enrich this stroke lattice with possible classifications of strokes and stroke combinations. The gesture recognizer uses a variant of the template matching approach described by Rubine (1991). This recognizes symbolic gestures such as lines, areas, points, arrows, and so on. The stroke lattice is then converted into an ink lattice which represents all of the possible interpretations of the user's ink as either symbolic gestures or handwritten words. The word lattice and ink lattice are integrated and assigned a combined meaning representation by the multimodal integration and understanding component (Johnston and Bangalore 2000; Johnston et al. 2002b). Because we implement this component using finite-state transducers, we refer to this component as the Multimodal Finite State Transducer (MMFST). The approach used in the MMFST component for integrating and interpreting multimodal inputs (Johnston et al. 2002a, 2002b) is an extension of the finite-state approach previously proposed (Bangalore and Johnston 2000; Johnston and Bangalore 2000, 2005). (See Section 3 for details.) This provides as output a lattice encoding all of the potential meaning representations assigned to the user's input. The meaning is represented in XML, facilitating parsing and logging by other system components. MMFST can receive inputs and generate outputs using multiple communication protocols, including the W3C EMMA standard for representation of multimodal inputs (Johnston et al. 2007). The meaning lattice is flattened to an $n$-best list and passed to a multimodal dialog manager (MDM) (Johnston et al. 2002b), which reranks the possible meanings in accordance with the current dialogue state. If additional information or confirmation is required, the MDM enters into a short information gathering dialogue with the user. Once a command or query is complete, it is passed to the multimodal generation component (MMGEN), which builds a multimodal score indicating a coordinated sequence of graphical actions and TTS prompts. This score is passed back to the MUI. The MUI then coordinates presentation of graphical content with synthetic speech output using the AT\&T Natural Voices TTS engine (Beutnagel et al. 1999). The subway route constraint solver (SUBWAY) is a backend server built for the prototype which identifies the best route between any two points in the city.

In the given example where the user says phone for these two restaurants while circling two restaurants (Figure 3(a) [A]), assume the speech recognizer returns the lattice in Figure 7 (Speech). The gesture recognition component also returns a lattice (Figure 7, Gesture) indicating that the user's ink is either a selection of two restaurants or a geographical area. The multimodal integration and understanding component (MMFST) combines these two input lattices into a lattice representing their combined meaning (Figure 7, Meaning). This is passed to the multimodal dialog manager (MDM) and from there to the MUI where it results in the display in Figure 3(a) [B] and coordinated TTS output.

The multimodal integration and understanding component utilizes a declarative multimodal grammar which captures both the structure and the interpretation of multimodal and unimodal commands. This formalism and its finite-state implementation for the MATCH system are explained in detail in Section 3.

This multimodal grammar is in part derived automatically by reference to an underlying ontology of the different kinds of objects in the application. Specific categories in the ontology, such as located_entity, are associated with templates and macros that are used to automatically generate the necessary grammar rules for the multimodal grammar and to populate classes in a class-based language model (Section 5). For example, in order to add support for a new kind of entity, for example, bars, a category bar is added to the ontology as a subtype of located_entity along with specification of the head nouns used for this new category, the attributes that apply to it, the symbol to use for it in the gesture representation, and a reference to the appropriate table to find bars 


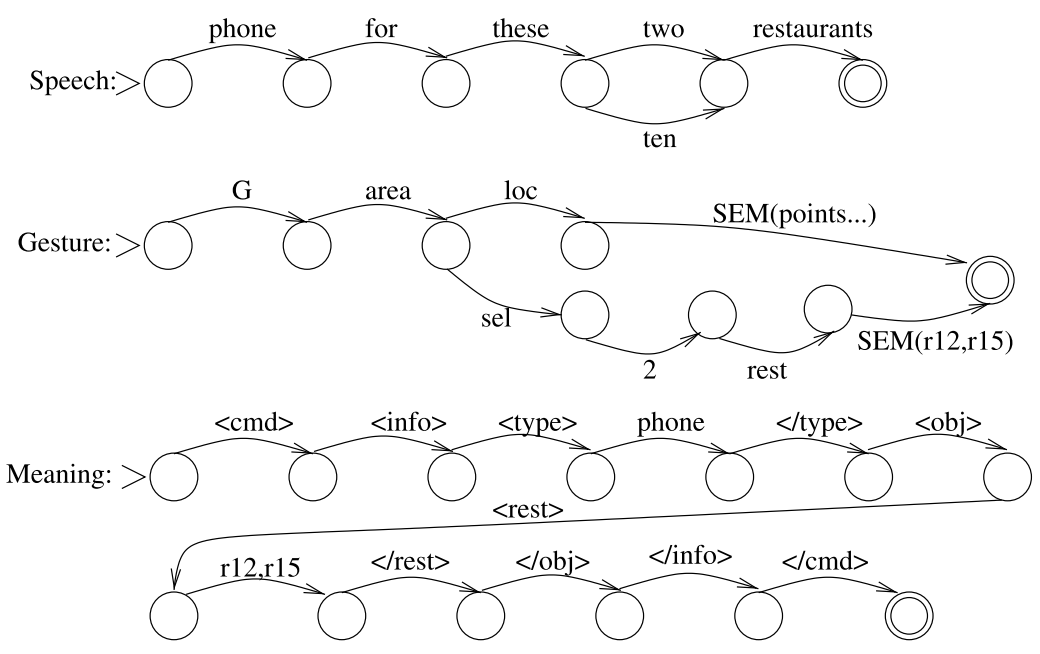

Figure 7

Multimodal example.

in the underlying application database. The appropriate multimodal grammar rules are then derived automatically as part of the grammar compilation process. Because the new entity type bar is assigned the ontology category located_entity, the grammar will automatically support deictic reference to bars with expressions such as this place in addition to the more specific this bar.

In the next section, we explain the data collection procedure we employed in order to evaluate the system and provide a test set for experimenting with different techniques for multimodal integration and understanding.

\subsection{Multimodal Data Collection}

A corpus of multimodal data was collected in a laboratory setting from a genderbalanced set of 16 first-time novice users. The subjects were AT\&T personnel with no prior knowledge of the system and no experience building spoken or multimodal systems. A total of 833 user interactions (218 multimodal/491 speech-only/124 penonly) resulting from six sample task scenarios involving finding restaurants of various types and getting their names, phones, addresses, or reviews, and getting subway directions between locations were collected and annotated.

Figure 8 shows the experimental set-up. Subjects interacted with the system in a soundproof room separated from the experimenter by one-way glass. Two video feeds were recorded, one from a scan converter connected to the system, the other from a camera located in the subject room, which captured a side-on view of the subject and the display. The system ran on a Fujitsu tablet computer networked to a desktop PC logging server located next to the experimenter. The subject's audio inputs were captured using both a close-talking headset microphone and a desktop microphone (which captured both user input and system audio).

As the user interacted with the system a multimodal log in XML format was captured on the logging server (Ehlen, Johnston, and Vasireddy 2002). The log contains a detailed record of the subject's speech and pen inputs and the system's internal processing steps and responses, with links to the relevant audio files and speech recognition lattices. 


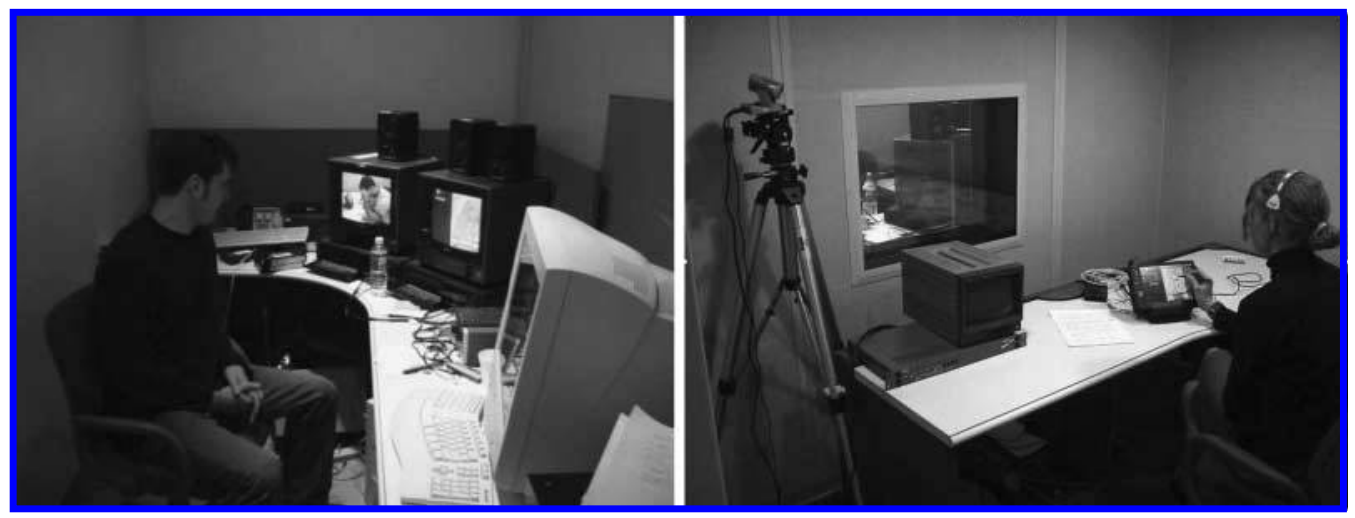

Figure 8

Experimenter and subject set-up.

The experimenter started out each subject with a brief tutorial on the system, showing them the pen and how to click on the display in order to turn on the microphone. The tutorial was intentionally vague and broad in scope so the subjects might overestimate the system's capabilities and approach problems in new ways. The experimenter then left the subject to complete, unassisted, a series of six sample task scenarios of varying complexity. These involved finding restaurants of various types and getting their names, phones, addresses, or reviews, and getting subway directions between locations. The task scenarios were presented in a GUI on the tablet next to the map display. In our pilot testing, we presented users with whole paragraphs describing scenarios. We found that users would often just rephrase the wording given in the paragraph, thereby limiting the utility of the data collection. Instead, in this data collection we presented what the user had to find as a table (Table 1). This approach elicited a broader range of inputs from users.

After completing the scenarios the user then completed an online questionnaire on the tablet regarding their experience with the system. This consisted of a series of Likert scale questions to measure user satisfaction (Walker, Passonneau, and Boland 2001). After the questionnaire the experimenter came into the experiment room and conducted an informal qualitative post-experiment feedback interview.

The next phase of the data collection process was to transcribe and annotate the users' input. Transcription is more complex for multimodal systems than for speechonly systems because the annotator needs not just to hear what the user said but also to see what they did. The browser-based construction of the multimodal user interface enabled us to rapidly build a custom version of the system which serves as an online multimodal annotation tool (Figure 9). This tool extends the approach described in Ehlen, Johnston, and Vasireddy (2002) with a graphical interface for construction of

Table 1

Example scenario.

Use MATCH to find the name, address, and phone number of a restaurant matching the following criteria:

Food Type Location

Vegetarian Union Square 


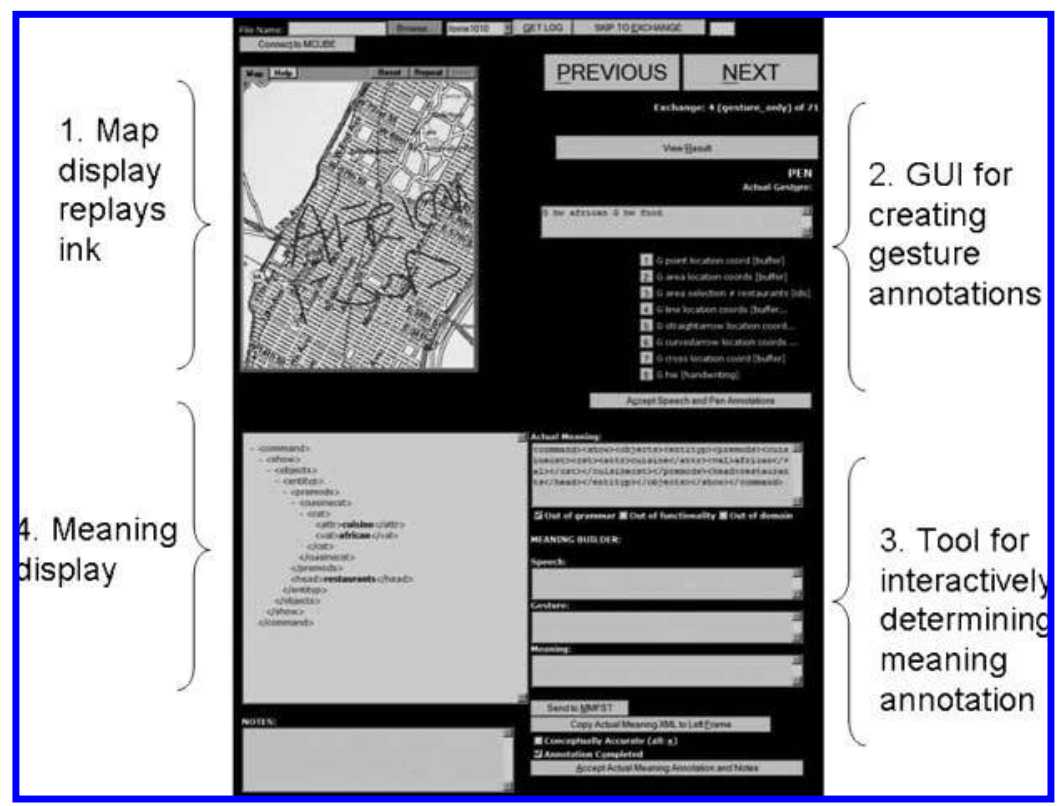

Figure 9

Multimodal $\log$ annotation tool.

gesture annotations and a tool for automatically deriving the meaning annotation for out-of-grammar examples. This tool allows the annotator to dynamically replay the users' inputs and system responses on the interactive map system itself, turn by turn, and add annotations to a multimodal log file, encoded in XML. The annotation utilizes the map component of the system (Figure 9(1)). It provides coordinated playback of the subject's audio with their electronic ink, enabling the user to rapidly annotate multimodal data without having to replay video of the interaction. The user interface of the multimodal log viewer provides fields for the annotator to transcribe the speech input, the gesture input, and the meaning. A series of buttons and widgets are provided to enable the annotator to rapidly and accurately transcribe the user's gesture and the appropriate meaning representation without having to remember the specifics of the gesture and meaning representations (Figure 9(2)).

After transcribing the speech and gesture, the annotator hits a button to confirm these, and they are recorded in the log and copied down to a second field used for annotating the meaning of the input (Figure 9(3)). It would be both time consuming and error-prone to have the annotator code in the meaning representation for each input by hand. Instead the multimodal understanding system is integrated into the multimodal annotation tool directly. The interface allows the annotator to adjust the speech and gesture inputs and send them through the multimodal understander until they get the meaning they are looking for (Figure 9(4)). When the multimodal understander returns multiple possibilities an $n$-best list is presented and the annotator hits the button next to the appropriate interpretation in order to select it as the annotated meaning. We found this to be a very effective method of annotating meaning, although it does require the annotator to have some knowledge of what inputs are acceptable to the system. In addition to annotating the speech, gesture, and meaning, annotators also checked off a series of flags indicating various properties of the exchange, such as whether the input was partial, whether there was a user error, and so on. The result of this effort was a 
corpus of 833 user interactions all fully annotated with speech, gesture, and meaning transcriptions.

\section{Multimodal Grammars and Finite-State Multimodal Language Processing}

One of the most critical technical challenges in the development of effective multimodal systems is that of enabling multimodal language understanding; that is, determining the user's intent by integrating and understanding inputs distributed over multiple modes. In early work on this problem (Neal and Shapiro 1991; Cohen 1991, 1992; Brison and Vigouroux 1993; Koons, Sparrell, and Thorisson 1993; Wauchope 1994), multimodal understanding was primarily speech-driven, ${ }^{1}$ treating gesture as a secondary dependent mode. In these systems, incorporation of information from the gesture input into the multimodal meaning is triggered by the appearance of expressions in the speech input whose reference needs to be resolved, such as definite and deictic noun phrases (e.g., this one, the red cube). Multimodal integration was essentially a procedural add-on to a speech or text understanding system.

Johnston et al. (1997) developed a more declarative approach where multimodal integration is modeled as unification of typed feature structures (Carpenter 1992) assigned to speech and gesture inputs. Johnston (1998a, 1998b) utilized techniques from natural language processing (unification-based grammars and chart parsers) to extend the unification-based approach and enable handling of inputs with more than one gesture, visual parsing, and more flexible and declarative encoding of temporal and spatial constraints. In contrast to the unification-based approaches, which separate speech parsing and multimodal integration into separate processing stages, Johnston and Bangalore $(2000,2005)$ proposed a one-stage approach to multimodal understanding in which a single grammar specified the integration and understanding of multimodal language. This avoids the complexity of interfacing between separate speech understanding and multimodal parsing components. This approach is highly efficient and enables tight coupling with speech recognition, because the grammar can be directly compiled into a cascade of finite-state transducers which can compose directly with lattices from speech recognition and gesture recognition components.

In this section, we explain how the finite-state approach to multimodal language understanding can be extended beyond multimodal input with simple pointing gestures made on a touchscreen (as in Johnston and Bangalore [2000, 2005]) to applications such as MATCH with complex gesture input combining freeform drawings with handwriting recognition. This involves three significant extensions to the approach: the development of a gesture representation language for complex pen input combining freehand drawing with selections and handwriting (Section 3.1); a new more scalable approach to abstraction over the specific content of gestures within the finite-state mechanism (Section 3.3); and a new gesture aggregation algorithm which enables robust handling of the integration of deictic phrases with a broad range of different selection gestures (Section 3.4). In Section 3.2, we illustrate the use of multimodal grammars for this application with a fragment of the multimodal grammar for MATCH and illustrate how this grammar is compiled into a cascade of finite-state transducers. Section 3.5 addresses the issue of temporal constraints on multimodal integration. In Section 3.6, we describe the multimodal dialog management mechanism used in the system and how

1 To be more precise, they are "verbal language"-driven, in that either spoken or typed linguistic expressions are the driving force of interpretation. 


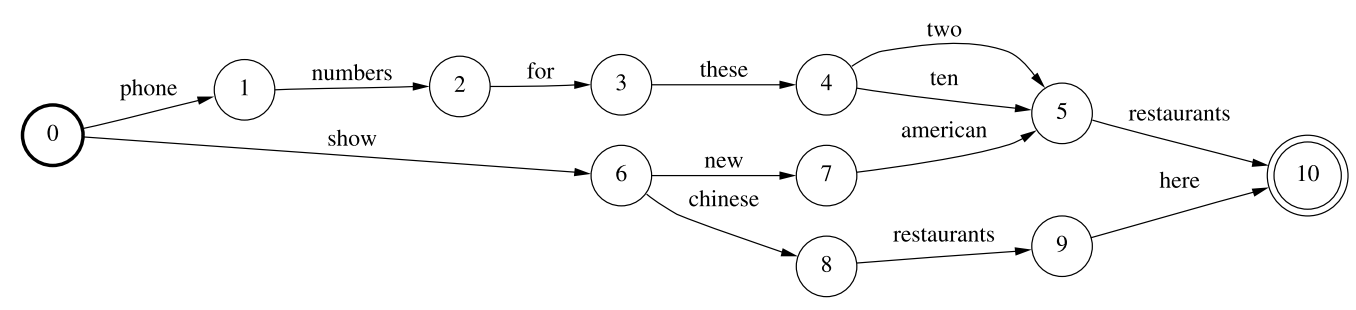

Figure 10

Speech lattice.

contextual resolution of deictic expressions is accounted for. In Section 3.7, we evaluate the performance of this approach to multimodal integration and understanding using the multimodal data collected as described in Section 2.2.

\subsection{Lattice Representations for Gesture and Meaning}

One of the goals of our approach to multimodal understanding is to allow for ambiguities and errors in the recognition of the individual modalities to be overcome through combination with the other mode (Oviatt 1999; Bangalore and Johnston 2000). To maximize the potential for error compensation, we maintain multiple recognition hypotheses by representing input modes as weighted lattices of possible recognition strings. For speech input, the lattice is a network of word hypotheses with associated weights. Figure 10 presents a simplified speech lattice from the MATCH application. ${ }^{2}$

Representation of Gesture. Like speech, gesture input can also be represented as a token stream, but unlike speech there is no pre-established tokenization of gestures (words of a gesture language) other than for handwritten words. We have developed a gesture representation language for pen input which enables representation of symbolic gestures such as areas, lines, and arrows, selection gestures, and handwritten words. This language covers a broad range of pen-based input for interactive multimodal applications and can easily be extended to new domains with different gesture symbols. Each gesture is represented as a sequence of symbols indicating different characteristics of the gesture. These symbol sequences can be concatenated in order to represent sequences of gestures and assembled into a lattice representation in order to represent a range of possible segmentations and interpretations of a sequence of ink strokes. In the MATCH system, when the user draws on the map, their ink points are captured along with information about potentially selected items, and these are passed to a gesture processing component. First, the electronic ink is rotated and scaled and broken into a lattice of strokes. This stroke lattice is processed by both gesture and handwriting recognizers to identify possible pen gestures and handwritten words in the ink stream. The results are combined with selection information to derive the gesture lattice representations presented in this section. The gesture recognizer uses a variant of the trained template matching approach described in Rubine (1991). The handwriting recognizer is neuralnetwork based. Table 2 provides the full set of eight gestures supported and the symbol sequences used to represent them in the gesture lattice.

2 The lattices in the actual system are weighted but for ease of exposition here we leave out weights in the figures. 
Table 2

Gesture inputs supported.

\begin{tabular}{|c|c|c|}
\hline Gesture & Example & Representation \\
\hline Area & & G area loc coords SEM \\
\hline Line & & $\mathrm{G}$ line loc coords SEM \\
\hline Point & & G point loc coord SEM \\
\hline Area Selection & & G area sel 1 rest SEM \\
\hline Point Selection & & G point sel 1 rest SEM \\
\hline Arrow & & G arrow base SEM head SEM angle SEM \\
\hline Question Mark & & G question_mark coords SEM \\
\hline Handwritten words & & G hw italian \\
\hline
\end{tabular}

For symbolic gestures and selections, the gesture symbol complexes have the basic form: G FORM MEANING (NUMBER TYPE) SEM. FORM indicates the physical form of the gesture, and has values such as area, point, line, and arrow. MEANING provides a rough characterization of the specific meaning of that form; for example, an area can be either a loc (location) or a sel (selection), indicating the difference between gestures which delimit a spatial location on the screen and gestures which select specific displayed icons. NUMBER and TYPE are only found with sel. They indicate the number of entities selected (1, 2, 3, many) and the specific type of entity (e.g., rest (restaurant) or thtr (theater)). The TYPE value mix is used for selections of entities of different types. Recognition of inputs as handwritten words is also encoded in the gesture lattice. These are indicated by the sequence G hw WORD. For example, if the user wrote phone number the gesture sequence would be $G$ hw phone $G$ hw number.

As an example, if the user draws an area on the screen which contains two restaurants (as in Figure 3(a) [A]), and the restaurants have associated identifiers $i d 1$ and $i d 2$, 
the gesture lattice will be as in Figure 11. The first two paths through this gesture lattice represent the ambiguity between the use of the gesture to indicate a spatial location versus a selection of objects on the screen. As defined in the subsequent multimodal grammar, if the speech is show me chinese restaurants in this neighborhood then the first path will be chosen. If the speech is tell me about these two restaurants then the second path will be chosen. The third path represents the recognition hypothesis from handwriting recognition that this is a handwritten $O$. If instead the user circles a restaurant and a theatre, the lattice would be as in Figure 12. If they say tell me about this theater, the third path will be taken. If they say tell me about these two, the fourth path will be taken. This allows for cases where a user circles several entities and selects a specific one by type.

The underlying ontology of the application domain plays a critical role in the handling of multimodal expressions. For example, if place in tell me about this place can refer to either a restaurant or a theatre, then it can be aligned with both gesture symbols in the multimodal grammar. The noun place is associated in the lexicon with a general type in the ontology: located_entity. When the multimodal grammar is compiled, by virtue of this type assignment, the expression this place is associated with gesture representations for all of the specific subtypes of located_entity in the ontology, such as restaurant and theater. The approach also extends to support deictic references to collections of objects of different types. For example, the noun building is associated in the lexicon with the type building. In the grammar these buildings is associated with the gesture type building. If the user selects a collection of objects of different types they are assigned the type building in the gesture lattice and so the expression these buildings will pick out that path. In the application domain of our prototype, where restaurants and theaters are the only selectable object types, we use a simpler ontology with a single general object type mix for collections of objects as in Figure 12, and this integrates with spoken phrases such as these places.

Representation of Meaning. Understanding multimodal language is about extracting the meaning from multimodal utterances. Although there continue to be endless debates in

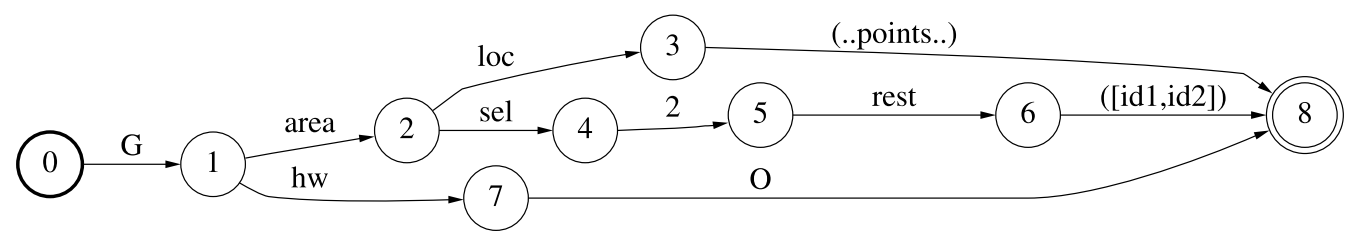

Figure 11

Gesture lattice G: Selection of two restaurants.

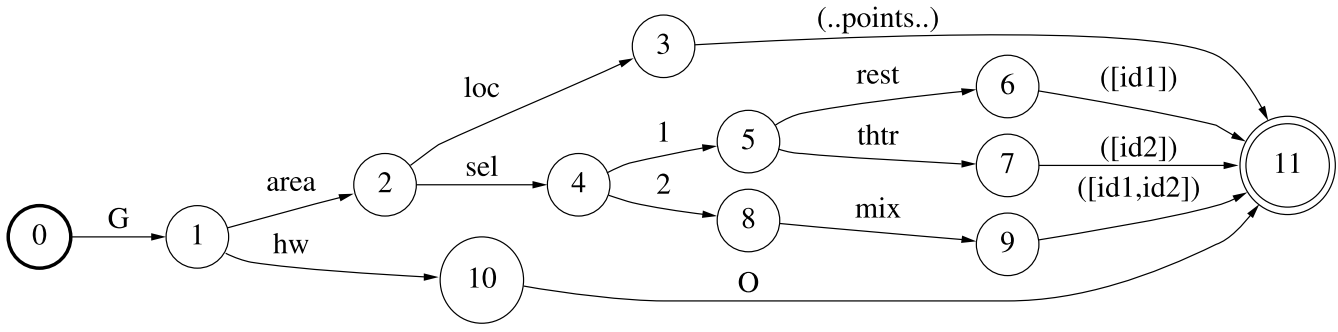

Figure 12

Gesture lattice G: Restaurant and theater. 


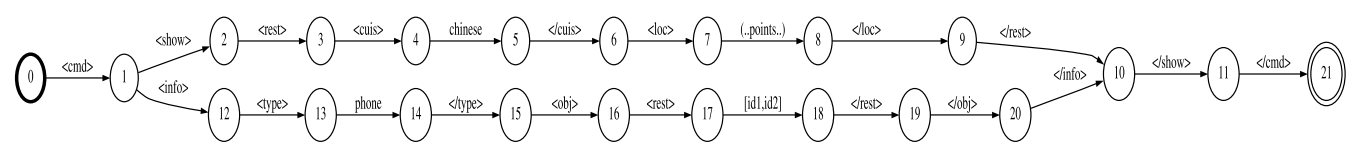

Figure 13

Meaning lattice.

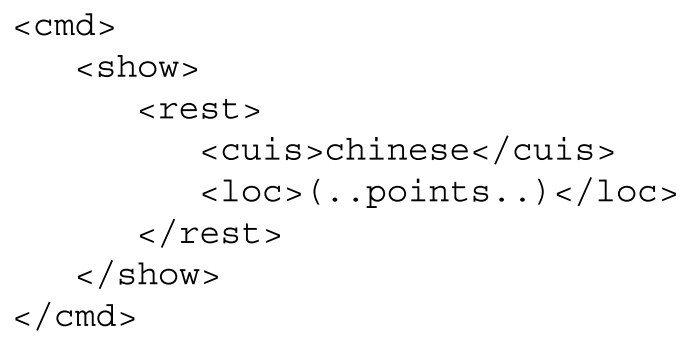

Figure 14

XML meaning representation.

linguistics, philosophy, psychology, and neuroscience on what constitutes the meaning of a natural language utterance (Jackendoff 2002), for the purpose of human-computer interactive systems, "meaning" is generally regarded as a representation that can be executed by an interpreter in order to change the state of the system.

Similar to the input speech and gesture representations, in our approach the output meaning is also represented in a lattice format. This enables compact representation of multiple possible interpretations of the user's inputs and allows for later stages of processing, such as the multimodal dialog manager, to use contextual information to rescore the meaning lattice. In order to facilitate logging and parsing by other components (dialog manager, backend servers), the meaning representation language is encoded in XML. ${ }^{3}$ The meaning lattice resulting from combination of speech and gesture is such that for every path through the lattice, the concatenation of symbols from that path will result in a well-formed XML expression which can be evaluated with respect to the underlying application semantics. In the city information application this includes elements such as $<$ show $>$ which contains a specification of a kind of restaurant to show, with elements <cuis $>$ (cuisine), $<$ loc $>$ (location), and so on. Figure 13 shows the meaning lattice that would result when the speech lattice (Figure 10) combines with the gesture lattice (Figure 11).

The first path through the lattice results from the combination of the speech string show chinese restaurants here with an area gesture. Concatenating the symbols on this path, we have the well-formed XML expression in Figure 14.

\subsection{Multimodal Grammars and Finite-State Understanding}

Context-free grammars have generally been used to encode the sequences of input tokens (words) in a language which are considered grammatical or acceptable for processing in a single input stream. In some cases grammar rules are augmented with operations used to simultaneously build a semantic representation of an utterance (Ades and

3 In our earlier work (Johnston and Bangalore 2000, 2005), we generated a predicate logic representation, for example: email([person(id1), organization(id2)]). 
Steedman 1982; Pollard and Sag 1994; van Tichelen 2004). Johnston and Bangalore (2000, 2005) present a multimodal grammar formalism which directly captures the relationship between multiple input streams and their combined semantic representation. The non-terminals in the multimodal grammar are atomic symbols. The multimodal aspects of the grammar become apparent in the terminals. Each terminal contains three components $W: G: M$ corresponding to the two input streams and one output stream, where $W$ is for the spoken language input stream, $G$ is for the gesture input stream, and $M$ is for the combined meaning output stream. These correspond to the three representations described in Section 3.1. The epsilon symbol $(\epsilon)$ is used to indicate when one of these is empty within a given terminal. In addition to the gesture symbols ( $G$ area loc ...), $G$ contains a symbol SEM used as a placeholder for specific content (see Section 3.3).

In Figure 15, we present a fragment of the multimodal grammar used for the city information application described in this article. This grammar is simplified for ease of exposition. The rules capture spoken, multimodal, and pen-only commands for showing restaurants (SHOW), getting information about them (INFO), requesting subway directions (ROUTE), and zooming the map (ZOOM).

As in Johnston and Bangalore $(2000,2005)$, this multimodal grammar is compiled into a cascade of finite-state transducers. Finite-state machines have been extensively applied to many aspects of language processing, including speech recognition (Riccardi, Pieraccini, and Bocchieri 1996; Pereira and Riley 1997), phonology (Kartunnen 1991; Kaplan and Kay 1994), morphology (Koskenniemi 1984), chunking (Abney 1991; Joshi and Hopely 1997; Bangalore 1997), parsing (Roche 1999), and machine translation (Bangalore and Riccardi 2000). Finite-state models are attractive mechanisms for language processing since they are (a) efficiently learnable from data; (b) generally effective for decoding; and (c) associated with a calculus for composing machines which allows for straightforward integration of constraints from various levels of language processing. Furthermore, software implementing the finite-state calculus is available for research purposes (Noord 1997; Mohri, Pereira, and Riley 1998; Kanthak and Ney 2004; Allauzen et al. 2007).

We compile the multimodal grammar into a finite-state device operating over two input streams (speech and gesture) and one output stream (meaning). The transition symbols of the FSA correspond to the terminals of the multimodal grammar. For the sake of illustration here and in the following examples we will only show the portion of the three-tape finite-state device which corresponds to the DEICNP rule in the grammar in Figure 15. The corresponding finite-state device is shown in Figure 16. This three-tape machine is then factored into two transducers: $\mathcal{R}: G \rightarrow W$ and $\mathcal{T}:(G \times W) \rightarrow M$. The $\mathcal{R}$ machine (e.g., Figure 17) aligns the speech and gesture streams through a composition with the speech and gesture input lattices $(G \circ(G: W \circ W))$. The result of this operation is then factored onto a single tape and composed with the $\mathcal{T}$ machine (e.g., Figure 18) in order to map these composite gesture-speech symbols into their combined meaning $\left(G_{-} W: M\right)$. Essentially the three-tape transducer is simulated by increasing the alphabet size by adding composite multimodal symbols that include both gesture and speech information. A lattice of possible meanings is derived by projecting on the output of G_W:M.

Because the speech and gesture inputs to multimodal integration and understanding are represented as lattices, this framework enables mutual compensation for errors (Johnston and Bangalore 2005); that is, it allows for information from one modality to be used to overcome errors in the other. For example, a lower confidence speech result may be selected through the integration process because it is semantically compatible with a higher confidence gesture recognition result. It is even possible for 


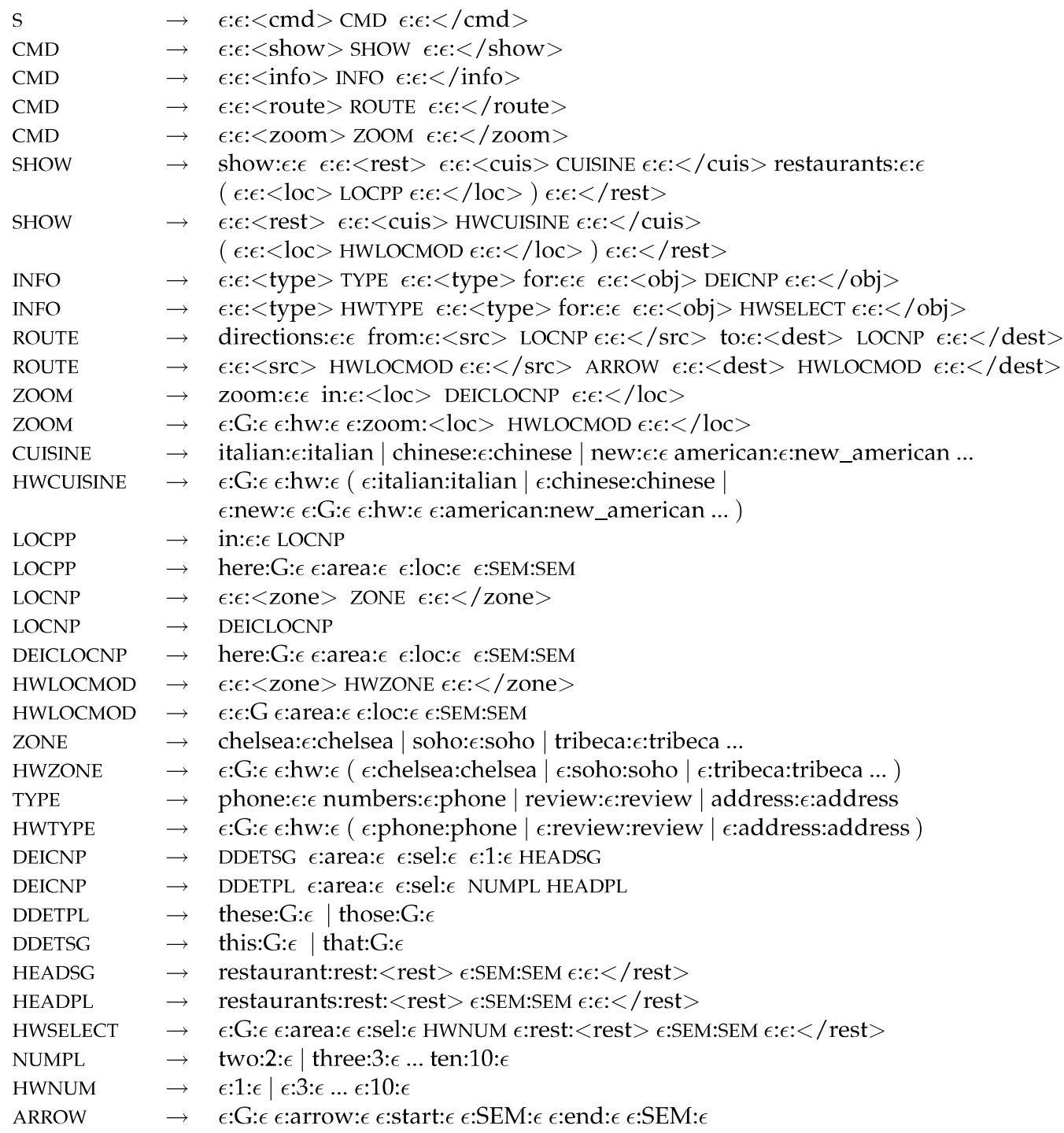

Figure 15

Multimodal grammar fragment.

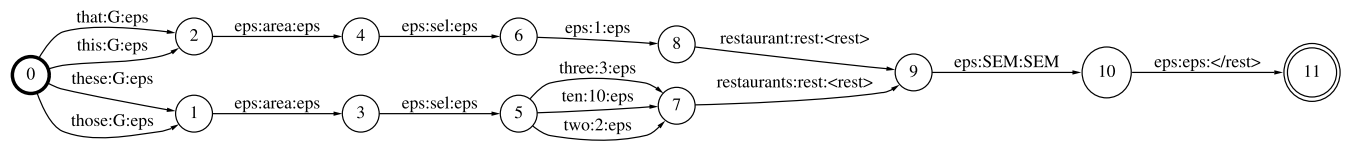

Figure 16

Multimodal three-tape FSA.

the system to overcome errors in both modalities within a single multimodal utterance. The multimodal composition process prunes out combinations of speech and gesture which are not semantically compatible and through combination of weights from the two different modalities it provides a ranking of the remaining semantically compatible combinations. This aspect of the approach is not the focus of this article and for ease 


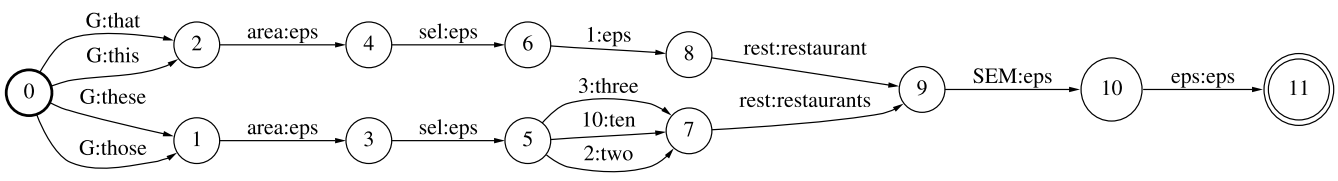

Figure 17

Gesture/speech alignment transducer.

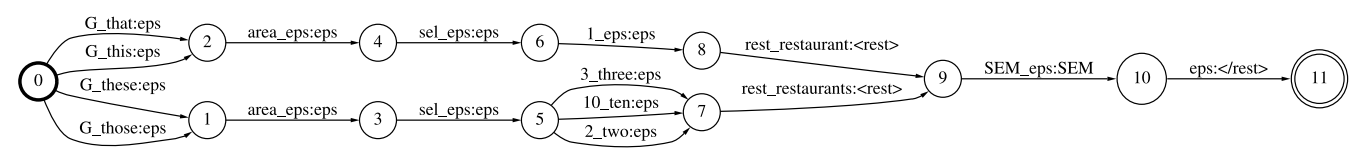

Figure 18

Gesture/speech to meaning transducer.

of exposition we have left out weights from the examples given. For the sake of completeness, we provide a brief description of the treatment of weights in the multimodal integration mechanism. The speech and gesture lattices contain weights. These weights are combined through the process of finite-state composition, so the finite-state device resulting from multimodal integration sums the weights from both the input lattices. In order to account for differences in reliability between the speech lattice weights and gesture lattice weights, the weights on the lattices are scaled according to a weighting factor $\lambda$ learned from held-out training data. The speech lattice is scaled by $\lambda: 0<\lambda<1$ and the gesture lattice by $1-\lambda$. Potentially this scaling factor could be dynamically adapted based on environmental factors and specific users' performance with the individual modes, though in the system described here the scaling factor was fixed for the duration of the experiment.

\subsection{Abstraction over Specific Gesture Content}

The semantic content associated with gesture inputs frequently involves specific information such as a sequence of map coordinates (e.g., for area gestures) or the identities of selected entities (e.g., restaurants or theaters). As part of the process of multimodal integration and understanding this specific content needs to be copied from the gesture stream into the resulting combined meaning. Within the finite-state mechanism, the only way to copy content is to have matching symbols on the gesture input and meaning output tapes. It is not desirable and in some cases infeasible to enumerate all of the different possible pieces of specific content (such as sequences of coordinates) so that they can be copied from the gesture input tape to the meaning output tape. This will significantly increase the size of the machine. In order to capture multimodal integration using finite-state methods, it is necessary to abstract over certain aspects of the gestural content.

We introduce here an approach to abstraction over specific gesture content using a number of additional finite-state operations. The first step is to represent the gesture input as a transducer $I: G$ where the input side contains gesture symbols and the specific content and the output side contains the same gesture symbols but a reserved symbol SEM appears in place of any specific gestural content such as lists of points or entity identifiers. The I:G transducer for the gesture lattice $G$ in Figure 11 is as shown in Figure 19. 


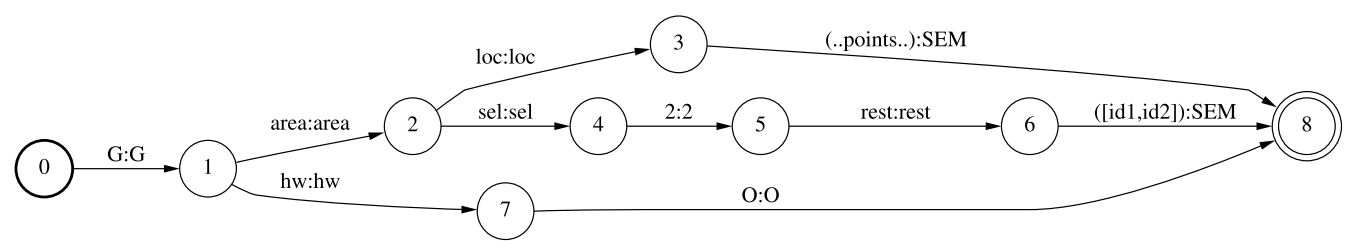

Figure 19

I:G transducer: Two restaurants.

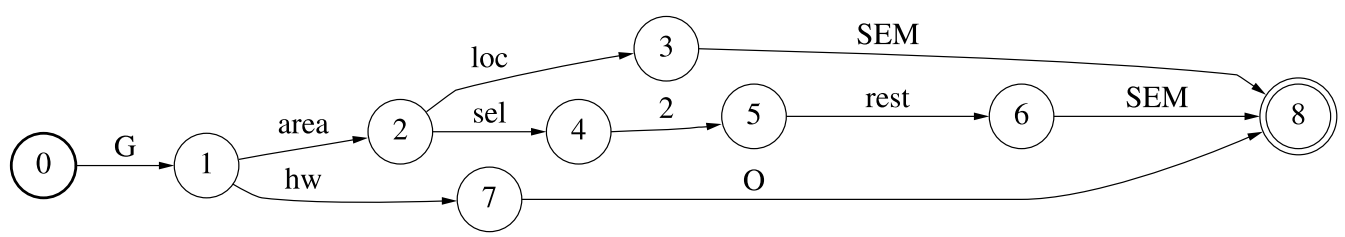

Figure 20

Gesture lattice G.

In any location in the multimodal grammar (Figure 15) and corresponding threetape finite-state device (Figure 16) where content needs to be copied from the gesture input into the meaning, the transition $\epsilon: S E M: S E M$ is used. In the $\mathcal{T}:(G \times W) \rightarrow M$ (Figure 17) transducer these transitions are labeled SEM_є:SEM.

For composition with the G:W gesture/speech alignment transducer (Figure 18) we take a projection of the output of the $I: G$ transducer. For the example $I: G$ transducer (Figure 19) the output projection $G$ is as shown in Figure 20. This projection operation provides the abstraction over the specific content.

After composing the $G$ and $W$ with $G: W$, factoring this transducer into an FSA $G_{-} W$ and composing it with $\mathcal{T}:(G \times W) \rightarrow M$, we are left with a transducer $G_{-} W: M$. This transducer combines a meaning lattice $M$ with a specification of the gesture and speech symbols and is used to determine the meaning of $G_{-} W$.

The next step is to factor out the speech information $(W)$, resulting in a transducer G:M which relates a meaning lattice $M$ to the gestures involved in determining those meanings $G$. This machine can be composed with the original $I: G$ transducer (I:Go $G: M)$, yielding a transducer $I: M$. The final step is to read off meanings from the $I: M$ transducer. For each path through the meaning lattice we concatenate symbols from the output $M$ side, unless the $M$ symbol is $S E M$ in which case we take the input $I$ symbol for that arc. Essentially, the I:G transducer provides an index back from the gesture symbol sequence associated with each meaning in the meaning lattice to the specific content associated with each gesture.

For our example case, if the speech these two restaurants is aligned with the gesture lattice (Figure 20) using $\mathcal{R}: G \rightarrow W$ (Figure 18) and the result is then factored and composed with $\mathcal{T}:(G \times W) \rightarrow M$ (Figure 17), the resulting $G_{-} W: M$ transducer is as in Figure 21. This is then factored in the G:M transducer Figure 22 and composed with $I: G$ (Figure 19), yielding the I:M transducer shown in Figure 23.

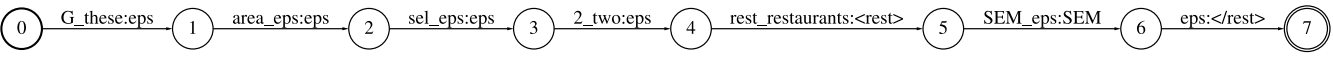

Figure 21

G_W:M transducer. 


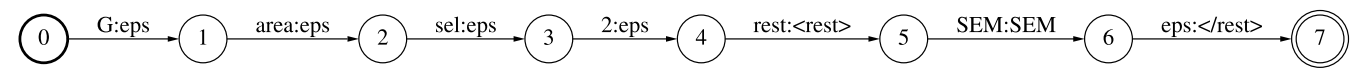

Figure 22

$G: M$ transducer.

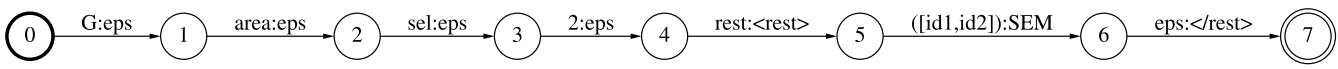

Figure 23

I:M transducer.

The meaning is generated by reading off and concatenating meaning symbols from the output of the I:M transducer, except for cases in which the output symbol is SEM, where instead the input symbol is taken. Alternatively, for all arcs in the I:M transducer where the output is $S E M$, the input and output symbols can be swapped (because the input label represents the value of the SEM variable), and then all paths in $M$ will be the full meanings with the specific content. For our example case this results in the following meaning representation: $<$ rest $>[r 12, r 15]</$ rest $>$. This example was only for the DEICNP subgrammar. With the full string phone numbers for these two restaurants the complete resulting meaning is: $\langle$ cmd $><$ info $><$ type $>$ phone $</$ type $><$ obj $\rangle<$ rest $\rangle$ $[$ r12,r15] $</$ rest $></$ obj $></$ info $></$ cmd $>$.

A critical advantage of this approach is that, because the gesture lattice itself is used to store the specific contents, the retrieval mechanism scales as the size and complexity of the gesture lattice increases. In the earlier approach more and more variable names are required as lattices increase in size, and in all places in the grammar where content is copied from gesture to meaning, arcs must be present for all of these variables. Instead here we leverage the fact that the gesture lattice itself can be used as a data structure from which the specific contents can be retrieved using the finite-state operation of composing I:G and G:M. This has the advantage that the algorithms required for abstracting over the specific contents and then reinserting the content are not required, and these operations are instead captured within the finite-state mechanism. One of the advantages of this representation of the abstraction is that it encodes not just the type of each gesture but also its position within the gesture lattice.

\subsection{Gesture Aggregation}

Johnston (2000) identifies problems involved in multimodal understanding and integration of deictic numeral expressions such as these three restaurants. The problem is that for a particular spoken phrase there are a multitude of different lexical choices of gesture and combinations of gestures that can be used to select the specified plurality of entities and all of these need to be integrated with the spoken phrase. For example, as illustrated in Figure 24, the user might circle all three restaurants with a single pen stroke, circle each in turn, or circle a group of two and group of one.

In the unification-based approach to multimodal parsing (Johnston 1998b), capturing all of these possibilities in the spoken language grammar significantly increases its size and complexity and any plural expression is made massively ambiguous. The suggested alternative in Johnston (2000) is to have the deictic numeral subcategorize for a plurality of the appropriate number and predictively apply a set of gesture combination rules in order to combine elements of gestural input into the appropriate pluralities. 


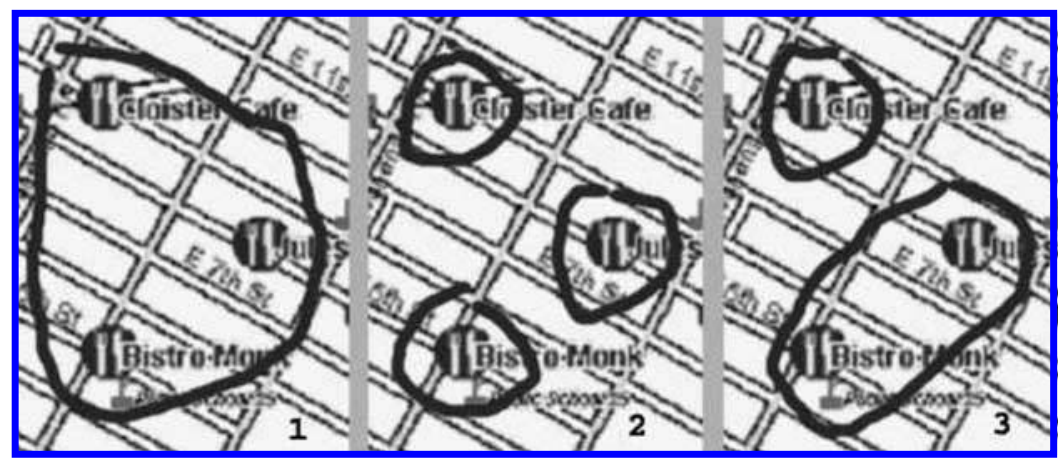

Figure 24

Multiple ways to select.

In the finite-state approach described here this can be achieved using a process we term gesture aggregation, which serves as a pre-processing phase on the gesture input lattice. A gesture aggregation algorithm traverses the gesture input lattice and adds new sequences of arcs which represent combinations of adjacent gestures of identical type. The operation of the gesture aggregation algorithm is described in pseudo-code in Algorithm 1. The function plurality() retrieves the number of entities in a selection gesture; for example, for a selection of two entities $g 1$, plurality $(g 1)=2$. The function type() yields the type of the gesture; for example rest for a restaurant selection gesture. The function specific_content() yields the specific IDs.

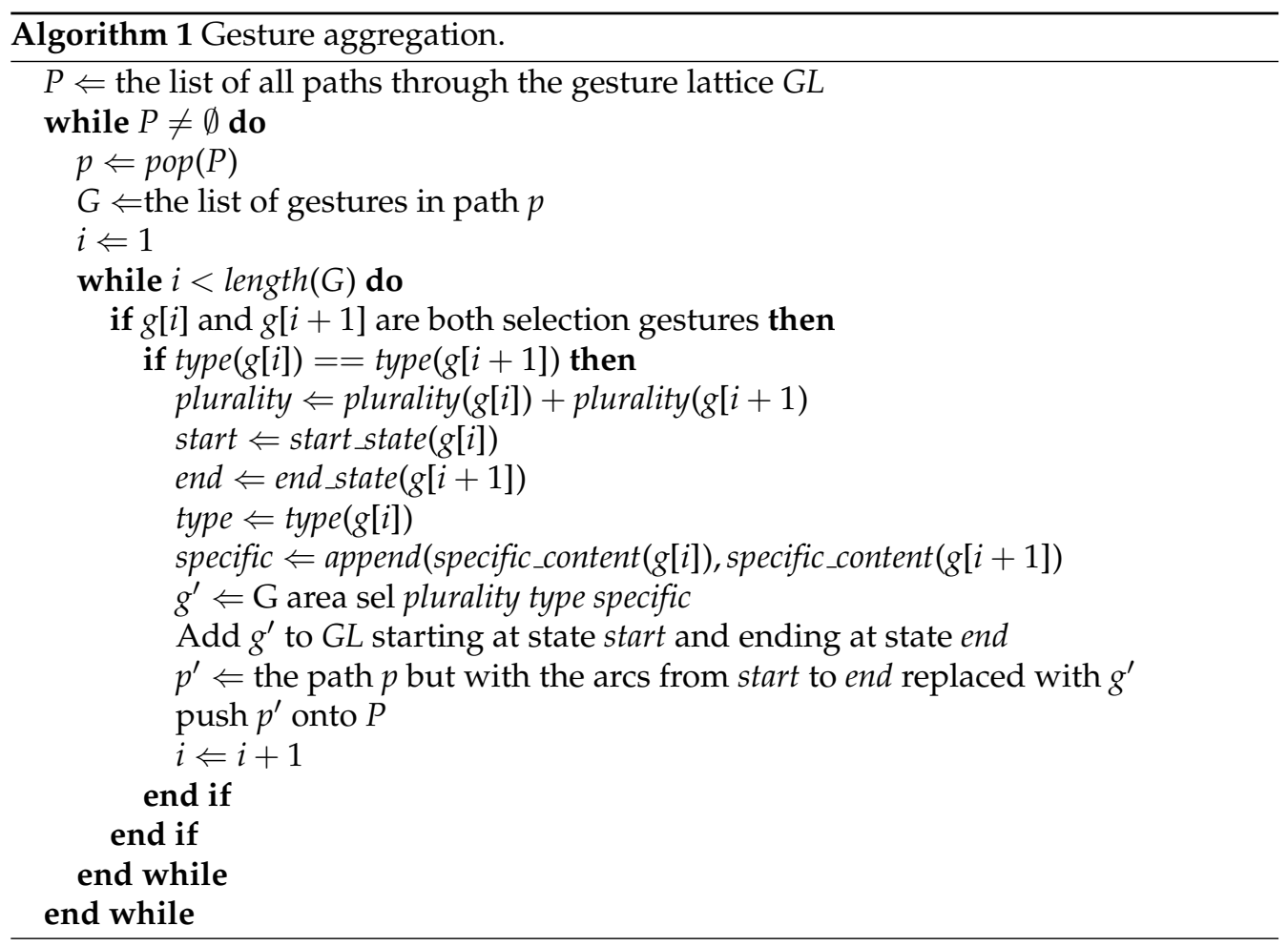


Essentially what this algorithm does is perform closure on the gesture lattice of a function which combines adjacent gestures of identical type. For each pair of adjacent gestures in the lattice which are of identical type, a new gesture is added to the lattice. This new gesture starts at the start state of the first gesture and ends at the end state of the second gesture. Its plurality is equal to the sum of the pluralities of the combining gestures. The specific content for the new gesture (lists of identifiers of selected objects) results from appending the specific contents of the two combining gestures. This operation feeds itself so that sequences of more than two gestures of identical type can be combined.

For our example case of three selection gestures on three different restaurants as in Figure 24(2), the gesture lattice before aggregation is as in Figure 25(a). After aggregation the gesture lattice is as in Figure 25(b). Three new sequences of arcs have been added. The first, from state 3 to state 8 , results from the combination of the first two gestures; the second, from state 14 to state 24, from the combination of the last two gestures; and the third, from state 3 to state 24, from the combination of all three gestures. The resulting lattice after the gesture aggregation algorithm has applied is shown in Figure 25(b). Note that minimization has been applied to collapse identical paths.

A spoken expression such as these three restaurants is aligned with the gesture symbol sequence $G$ area sel 3 rest SEM in the multimodal grammar. This will be able to combine not just with a single gesture containing three restaurants but also with our example gesture lattice, since aggregation adds the path: G area sel 3 rest [id1,id2,id3].

We term this kind of aggregation type specific aggregation. The aggregation process can be extended to support type non-specific aggregation for cases where users refer to sets of objects of mixed types and select them using multiple gestures. For example in the case where the user says tell me about these two and circles a restaurant and then a theater, non-type specific aggregation applies to combine the two gestures into an aggregate of mixed type $G$ area sel 2 mix $[(i d 1, i d 2)]$ and this is able to combine with these two. For applications with a richer ontology with multiple levels of hierarchy, the type non-specific aggregation should assign to the aggregate to the lowest common subtype of the set of entities being aggregated. In order to differentiate the original sequence of gestures that the user made from the aggregate, paths added through aggregation are assigned additional cost.

Figure 25(c) shows how these new processes of gesture abstraction and aggregation integrate into the overall finite-state multimodal language processing cascade. Aggregation applies to the $I: G$ representation of the gesture. A projection $G$ on the $I: G$ is composed with the gesture/speech alignment transducer $\mathcal{R}: G \rightarrow W$, then the result is composed with the speech lattice. The resulting $G: W$ transducer is factored into an FSA with a composite alphabet of symbols. This is then composed with the $\mathcal{T}:(G \times W) \rightarrow M$ yielding a result transducer $G_{-} W: M$. The speech is factored out of the input yielding $G: M$ which can then be composed with $I: G$, yielding a transducer $I: M$ from which the final meanings can be read.

\subsection{Temporal Constraints on Multimodal Integration}

In the approach taken here, temporal constraints for speech and gesture alignment are not needed within the multimodal grammar itself. Bellik $(1995,1997)$ provides examples indicating the importance of precise temporal constraints for proper interpretation of multimodal utterances. Critically, though, Bellik's examples involve not single 


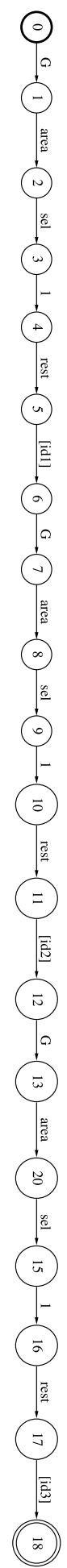

(a)

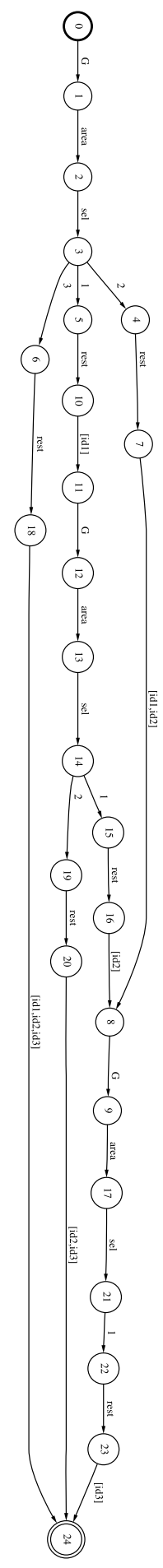

(b)

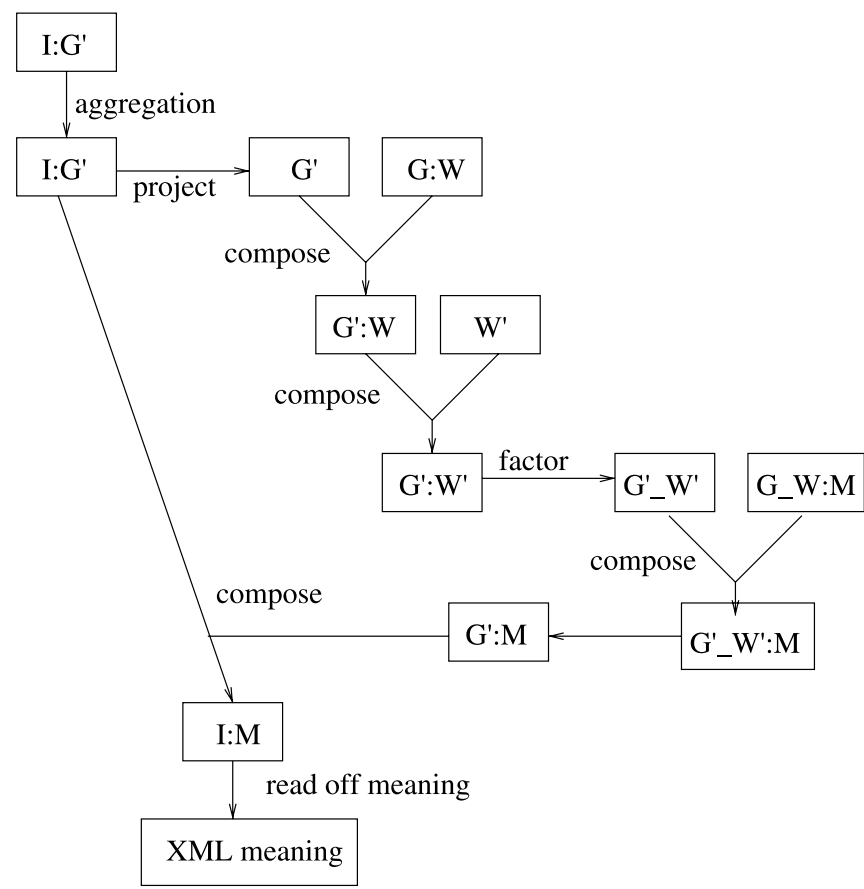

Figure 25

(a) Three gestures. (b) Aggregated lattice. (c) Multimodal language processing cascade. 
multimodal utterances but sequences of two utterances. ${ }^{4}$ The multimodal integration mechanism and multimodal grammars described herein enumerate the content of single turns of user input, be they unimodal or multimodal. The multimodal integration component and multimodal grammars are not responsible for combination of content from different modes that occur in separate dialog turns. This is treated as part of dialog management and reference resolution. Temporal constraints do, however, play a role in segmenting parallel multimodal input streams into single user turns. This is one of the functions of the multimodal understanding component. In order to determine which gestures and speech should be considered part of a single user utterance, a dynamic timeout adaptation mechanism was used. In initial versions of the system, fixed timeout intervals were used on receipt of input from one modality to see if the input is in fact unimodal or whether input in the other modality is forthcoming. In pilot studies we determined that the system latency introduced by these timeouts could be significantly reduced by making the timeouts sensitive to activity in the other mode. In addition to messages containing the results of speech recognition and gesture processing, we instrumented the multimodal understanding component (MMFST) to receive events indicating when the pen first touches the screen (pen-down event) and when the clickto-speak button is pressed (click-to-speak event). When the MMFST component receives a speech lattice, if a gesture lattice has already been received then the two lattices are processed immediately as a multimodal input. If gesture has not yet been received and there is no pen-down event, the multimodal component waits for a short timeout interval before interpreting the speech as a unimodal input. If gesture has not been received, but there has been a pen-down event, the multimodal component will wait for a longer timeout period for the gesture lattice message to arrive. Similarly, when gesture is received, if the speech lattice has already been received the two are integrated immediately. If speech has not yet arrived, and there was no click-to-speak event, then the system will wait for a short timeout before processing the gesture lattice as unimodal input. If speech has not yet arrived but the click-to-speak event has been received then the component will wait for the speech lattice to arrive for a longer timeout period. Longer timeouts are used instead of waiting indefinitely to account for cases where the speech or gesture processing does not return a result. In pilot testing we determined that with the adaptive mechanism the short timeouts could be kept as low as a second or less, significantly reducing system latency for unimodal inputs. With the non-adaptive mechanism we required timeouts of as much as two to three seconds. For the longer timeouts we found 15 seconds to be an appropriate time period. A further extension of this approach would be to make the timeout mechanism adapt to specific users, since empirical studies have shown that users tend to fall into specific temporal integration patterns (Oviatt, DeAngeli, and Kuhn 1997).

The adaptive timeout mechanism could also be used with other speech activation mechanisms. In an "open microphone" setting where there is no explicit click-to-speak event, voice activity detection could be used to signal that a speech event is forthcoming. For our application we chose a "click-to-speak" strategy over "open microphone" because it is more robust to noise and mobile multimodal interfaces are intended for use in environments subject to noise. The other alternative, "click-and-hold," where the user has to hold down a button for the duration of their speech, is also problematic because it limits the ability of the user to use pen input while they are speaking.

4 See Johnston and Bangalore (2005) for a detailed explanation. 


\subsection{Multimodal Dialog Management and Contextual Resolution}

The multimodal dialog manager (MDM) is based on previous work on speech-act based models of dialog (Rich and Sidner 1998; Stent et al. 1999). It uses a Java-based toolkit for writing dialog managers that is similar in philosophy to TrindiKit (Larsson et al. 1999). It includes several rule-based processes that operate on a shared state. The state includes system and user intentions and beliefs, a dialog history and focus space, and information about the speaker, the domain, and the available modalities. The processes include interpretation, update, selection, and generation.

The interpretation process takes as input an $n$-best list of possible multimodal interpretations for a user input from the MMFST. It rescores them according to a set of rules that encode the most likely next speech act given the current dialogue context, and picks the most likely interpretation from the result. The update process updates the dialogue context according to the system's interpretation of user input. It augments the dialogue history, focus space, models of user and system beliefs, and model of user intentions. It also alters the list of current modalities to reflect those most recently used by the user.

The selection process determines the system's next move(s). In the case of a command, request, or question, it first checks that the input is fully specified (using the domain ontology, which contains information about required and optional roles for different types of actions); if it is not, then the system's next move is to take the initiative and start an information-gathering subdialogue. If the input is fully specified, the system's next move is to perform the command or answer the question; to do this, MDM communicates directly with the UI.

The generation process performs template-based generation for simple responses and updates the system's model of the user's intentions after generation. A text planning component (TEXTPLAN) is used for more complex generation, such as the generation of comparisons (Walker et al. 2002, 2004).

In the case of a navigational query, such as the example in Section 2, MDM first receives a route query in which only the destination is specified: How do I get to this place?. In the selection phase it consults the domain ontology and determines that a source is also required for a route. It adds a request to query the user for the source to the system's next moves. This move is selected and the generation process selects a prompt and sends it to the TTS component. The system asks Where do you want to go from?. If the user says or writes 25th Street and 3rd Avenue then the MMFST will assign this input two possible interpretations: either this is a request to zoom the display to the specified location or it is an assertion of a location. Because the MDM dialogue state indicates that it is waiting for an answer of the type location, MDM reranks the assertion as the most likely interpretation. A generalized overlay process (Alexandersson and Becker 2001) is used to take the content of the assertion (a location) and add it into the partial route request. The result is determined to be complete. The UI resolves the location to map coordinates and passes on a route request to the SUBWAY component.

We found this traditional speech-act based dialogue manager worked well for our multimodal interface. Critical in this was our use of a common semantic representation across spoken, gestured, and multimodal commands. The majority of the dialogue rules operate in a mode-independent fashion, giving users flexibility in the mode they choose to advance the dialogue.

One of the roles of the multimodal dialog manager is to handle contextual resolution of deictic expressions. Because they can potentially be resolved either by integration with a gesture, or from context, deictic expressions such as this restaurant are 
ambiguous in the multimodal grammar. There will be one path through the grammar where this expression is associated with a sequence of gesture symbols, such as $G$ area selection 1 rest $r 123$, and another where it is not associated with any gesture symbols and assigned a semantic representation which indicates that it must be resolved from context: $<$ rest $><$ discourseref $></$ discourseref $></$ rest $>$. If at the multimodal understanding stage there is a gesture of the appropriate type in the gesture lattice, then the first of these paths will be chosen and the identifier associated with the gesture will be added to the semantics during the multimodal integration and understanding process: $<$ rest $>r 123</$ rest $>$. If there is no gesture, then this restaurant will be assigned the semantic representation $<$ rest $><$ discourseref $></$ discourseref $\rangle</$ rest $\rangle$ and the dialog manager will attempt to resolve the gesture from the dialog context. The update process in the multimodal dialog manager maintains a record in the focus space of the last mention of entities of each semantic type, and the last mentioned entity. When the interpretation process receives a semantic representation containing the marker $<$ rest $><$ discourseref $></$ discourseref $></$ rest $>$ it replaces $<$ discourseref $></$ discourseref $>$ with the identifier of the last-mentioned entity of the type restaurant.

Cases where the gesture is a low-confidence recognition result, in fact, where the gesture is spurious and not an intentional input, are handled using back-offs in the multimodal grammar as follows: In the multimodal grammar, productions are added which consume a gesture from the gesture lattice, but assign the semantics $<$ rest $><$ discourseref $></$ discourseref $></$ rest $>$. Generally these are assigned a higher cost than paths through the model where the gesture is meaningful, so that these backoff paths will only be chosen if there is no alternative. In practice for speech and pen systems of the kind described here, we have found that spurious gestures are uncommon, though they are likely to be considerably more of a problem for other kinds of modalities, such as freehand gesture recognized using computer vision.

\subsection{Experimental Evaluation}

To determine the baseline performance of the finite-state approach to multimodal integration and understanding, and to collect data for the experiments on multimodal robustness described in this article, we collected and annotated a corpus of multimodal data as described in Section 2.2. To enable this initial experiment and data collection, because no corpus data had already been collected, to bootstrap the process we initially used a handcrafted multimodal grammar using grammar templates combined with data from the underlying application database. As shown in Figure 26, the multimodal grammar can be used to create language models for ASR, align the speech and gesture results from the respective recognizers, and transform the multimodal utterance to a meaning representation. All these operations are achieved using finite-state transducer operations.

For the 709 inputs that involve speech (491 unimodal speech and 218 multimodal) we calculated the speech recognition accuracy (word and sentence level) for results using the grammar-based language model projected from the multimodal grammar. We also calculated a series of measures of concept accuracy on the meaning representations resulting from taking the results from speech recognition and combining them with the gesture lattice using the gesture speech alignment model, and then the multimodal understanding model. The concept accuracy measures: Concept Sentence Accuracy, Predicate Sentence Accuracy, and Argument Sentence Accuracy are explained subsequently. 


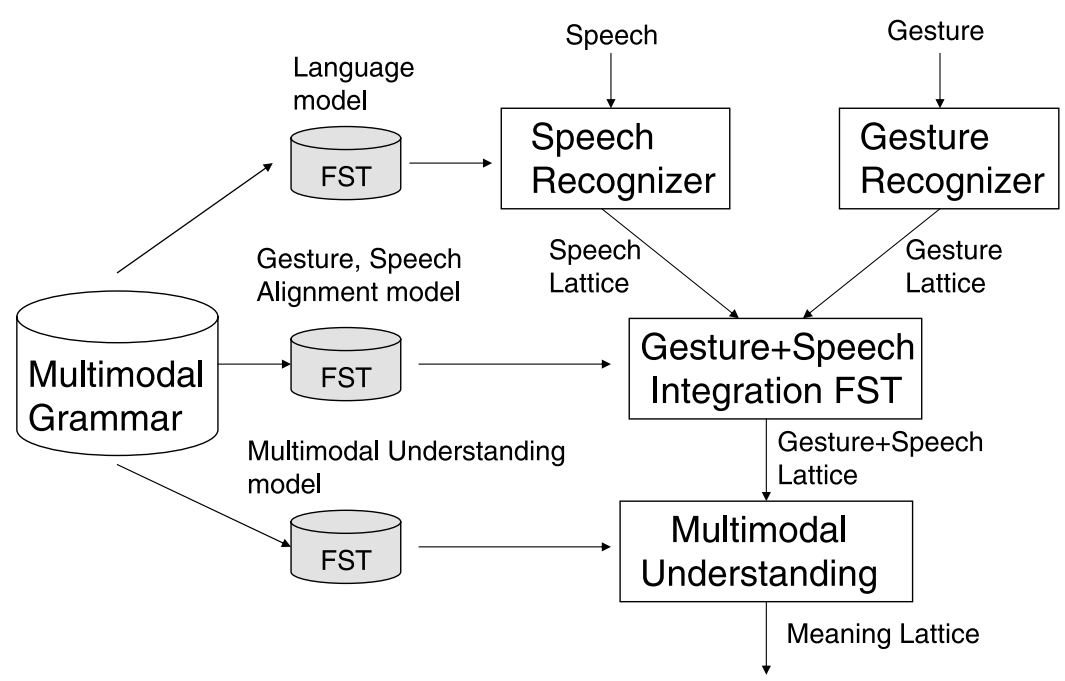

Figure 26

Multimodal grammar compilation for different processes of MATCH.

The hierarchically-nested XML representation described in Section 3.1 is effective for processing by the backend application, but is not well suited for the automated determination of the performance of the language understanding mechanism. We developed an approach, similar to Ciaramella (1993) and Boros et al. (1996), in which the meaning representation, in our case XML, is transformed into a sorted flat list of attribute-value pairs indicating the core contentful concepts of each command. The attribute-value meaning representation normalizes over multiple different XML representations which correspond to the same underlying meaning. For example, phone and address and address and phone receive different XML representations but the same attribute-value representation. For the example phone number of this restaurant, the XML representation is as in Figure 27, and the corresponding attribute-value representation is as in Figure 28.

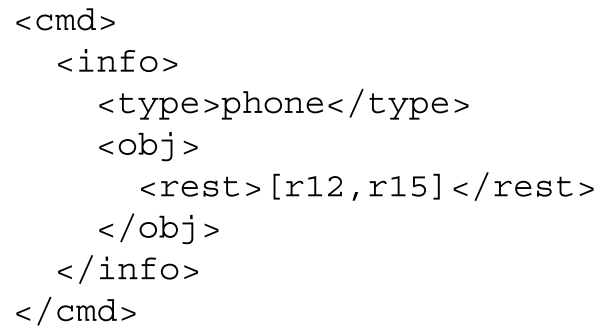

Figure 27

XML meaning representation.

cmd:info type:phone object:selection.

Figure 28

Attribute-value meaning representation. 
Table 3

ASR and concept accuracy for the grammar-based finite-state approach (10-fold).

\begin{tabular}{lll}
\hline Speech recognition & Word accuracy & $41.6 \%$ \\
& Sentence accuracy & $38.0 \%$ \\
Understanding & Concept sentence accuracy & $50.7 \%$ \\
& Predicate accuracy & $67.2 \%$ \\
& Argument accuracy & $52.8 \%$
\end{tabular}

This transformation of the meaning representation allows us to calculate the performance of the understanding component using string-matching metrics parallel to those used for speech recognition accuracy. Concept Sentence Accuracy measures the number of user inputs for which the system got the meaning completely right. ${ }^{5}$ Predicate Sentence Accuracy measures whether the main predicate of the sentence was correct (similar to call type in call classification). Argument Sentence Accuracy is an exact string match between the reference list of arguments and the list of arguments identified for the command. Note that the reference and hypothesized argument sequences are lexicographically sorted before comparison so the order of the arguments does not matter. We do not utilize the equivalent of word accuracy on the concept token sequence. The concept-level equivalent of word accuracy is problematic because it can easily be manipulated by increasing or decreasing the number of tokens used in the meaning representation.

To provide a baseline for the series of techniques explored in the rest of the article, we performed recognition and understanding experiments on the same 10 partitions of the data as in Section 4. The numbers are all averages over all 10 partitions. Table 3 shows the speech recognition accuracy using the grammar-based language model projected from the multimodal grammar. It also shows the concept accuracy results for the multimodal-grammar-based finite-state approach to multimodal understanding.

The multimodal grammars described here provide an expressive mechanism for quickly creating language processing capabilities for multimodal interfaces supporting input modes such as speech and pen, but like other approaches based on handcrafted grammars, multimodal grammars are brittle with respect to extra-grammatical or erroneous input. The language model directly projected from the speech portion of the hand-crafted multimodal grammar is not able to recognize any strings that are not encoded in the grammar. In our data, $62 \%$ of user's utterances were out of the multimodal grammar, a major problem for recognition (as illustrated in Table 3). The poor ASR performance has a direct impact on concept accuracy. The fact that the score for concept sentence accuracy is higher than that for sentence accuracy is not unexpected since recognition errors do not always result in changes in meaning and also to a certain extent the grammar-based language model will force fit out-of-grammar utterances to similar in-grammar utterances.

\section{Robustness in Multimodal Language Processing}

A limitation of grammar-based approaches to (multimodal) language processing is that the user's input is often not covered by the grammar and hence fails to receive an interpretation. This issue is present in grammar-based speech-only dialog systems

5 This metric is called Sentence Understanding in Ciaramella (1993). 
as well. The lack of robustness in such systems is due to limitations in (a) language modeling and (b) understanding of the speech recognition output.

The brittleness of using a grammar as a language model is typically alleviated by building SLMs that capture the distribution of the user's interactions in an application domain. However, such SLMs are trained on large amounts of spoken interactions collected in that domain-a tedious task in itself, in speech-only systems, but an often insurmountable task in multimodal systems. The problem we face is how to make multimodal systems more robust to disfluent or unexpected multimodal language in applications for which little or no training data is available. The reliance on multimodal grammars as a source of data is inevitable in such situations. In Section 5, we explore and evaluate a range of different techniques for building effective SLMs for spoken and multimodal systems under constraints of limited training data. The techniques are presented in the context of SLMs, since spoken language interaction tends to be a dominant mode in our application and has higher perplexity than the gesture interactions. However, most of these techniques can also be applied to improve the robustness of the gesture recognition component in applications with higher gesture language perplexity.

The second source of brittleness in a grammar-based multimodal/unimodal interactive system is in the assignment of meaning to the multimodal output. The grammar typically encodes the relation between the multimodal inputs and their meanings. The assignment of meaning to a multimodal output is achieved by parsing the utterance using the grammar. In a grammar-based speech-only system, if the language model of ASR is derived directly from the grammar, then every ASR output can be parsed and assigned a meaning by the grammar. However, using an SLM results in ASR outputs that may not be parsable by the grammar and hence cannot be assigned a meaning by the grammar. Robustness in such cases is achieved by either (a) modifying the parser to accommodate for unparsable substrings in the input (Ward 1991; Dowding et al. 1993; Allen et al. 2001) or (b) modifying the meaning representation to make it learnable as a classification task using robust machine learning techniques as is done in large scale human-machine dialog systems (e.g., Gorin, Riccardi, and Wright 1997).

In our grammar-based multimodal system, the grammar serves as the speechgesture alignment model and assigns a meaning representation to the multimodal input. Failure to parse a multimodal input implies that the speech and gesture inputs are not fused together and consequently may not be assigned a meaning representation. In order to improve robustness in multimodal understanding, more flexible mechanisms must be employed in the integration and the meaning-assignment phases. In Section 6, we explore and evaluate approaches that transform the multimodal input so as to be parsable by the multimodal grammar, as well as methods that directly map the multimodal input to the meaning representation without the use of the grammar. We again present these approaches in the context of transformation of the ASR output, but they are equally applicable to gesture recognition outputs. Transformation of the multimodal inputs so as to be parsable by the multimodal grammar directly improves robustness of multimodal integration and understanding.

Although some of the techniques presented in the next two sections are known in the literature, they have typically been applied in the context of speech-only dialog systems and on different application domains. As a result, comparing the strengths and weaknesses of these techniques is very difficult. By evaluating them on the MATCH domain, we are able to compare and extend these techniques for robust multimodal understanding. Other factors such as contextual information including dialog context, graphical display context, geographical context, as well as meta-information such as user preferences and profiles, can be used to further enhance the robustness of a 
multimodal application. However, here we focus on techniques for improving robustness of multimodal understanding that do not rely on such factors.

\section{Robustness of Language Models for Speech Recognition}

The problem of speech recognition can be succinctly represented as a search for the most likely word sequence $\left(w^{*}\right)$ through the network created by the composition of a language of acoustic observations $(O)$, an acoustic model which is a transduction from acoustic observations to phone sequences $(A)$, a pronunciation model which is a transduction from phone sequences to word sequences $(L)$, and a language model acceptor $(G)$ (Pereira and Riley 1997) (Equation 2). The language model acceptor encodes the (weighted) word sequences permitted in an application.

$$
w^{*}=\underset{w}{\operatorname{argmax}} \pi_{2}(O \circ A \circ L \circ G)(w)
$$

Typically, $G$ is built using either a hand-crafted grammar or using a statistical language model derived from a corpus of sentences from the application domain. Although a grammar could be written so as to be easily portable across applications, it suffers from being too prescriptive and has no metric for the relative likelihood of users' utterances. In contrast, in the data-driven approach a weighted grammar is automatically induced from a corpus and the weights can be interpreted as a measure of the relative likelihoods of users' utterances. However, the reliance on a domain-specific corpus is one of the significant bottlenecks of data-driven approaches, because collecting a corpus specific to a domain is an expensive and time-consuming task, especially for multimodal applications.

In this section, we investigate a range of techniques for producing a domain-specific corpus using resources such as a domain-specific grammar as well as an out-of-domain corpus. We refer to the corpus resulting from such techniques as a domain-specific derived corpus in contrast to a domain-specific collected corpus. We are interested in techniques that would result in corpora such that the performance of language models trained on these corpora would rival the performance of models trained on corpora collected for a specific domain. We investigate these techniques in the context of MATCH. We use the notation $C_{d}$ for the corpus, $\lambda_{d}$ for the language model built using the corpus $C_{d}$, and $G_{\lambda_{d}}$ for the language model acceptor representation of the model $\lambda_{d}$ which can be used in Equation (2).

\subsection{Language Model Using In-Domain Corpus}

We used the MATCH domain corpus from the data collection to build a class-based trigram language model $\left(\lambda_{M A T C H}\right)$ using the 709 multimodal and speech-only utterances as the corpus $\left(C_{M A T C H}\right)$. We used the names of cuisine types, areas of interest, points of interest, and neighborhoods as classes when building the trigram language model. The trigram language model is represented as a weighted finite-state acceptor (Allauzen, Mohri, and Roark 2003) for speech recognition purposes. The performance of this model serves as the point of reference to compare the performance of language models trained on derived corpora. 


\subsection{Grammar as Language Model}

The multimodal context-free grammar (CFG; a fragment is presented in Section 2 and a larger fragment is shown in Section 3.2) encodes the repertoire of language and gesture commands allowed by the system and their combined interpretations. The CFG can be approximated by a finite state machine (FSM) with arcs labeled with language, gesture, and meaning symbols, using well-known compilation techniques (Nederhof 1997). Selecting the language symbol of each arc (projecting the FSM on the speech component) results in an FSM that can be used as the language model acceptor $\left(G_{\text {gram }}\right)$ for speech recognition. Note that the resulting language model acceptor is unweighted if the grammar is unweighted and suffers from not being robust to language variations in users' input. However, due to the tight coupling of the grammars used for recognition and interpretation, every recognized string can be assigned a meaning representation (though it may not necessarily be the intended interpretation).

\subsection{Grammar-Based $n$-gram Language Model}

As mentioned earlier, a hand-crafted grammar typically suffers from the problem of being too restrictive and inadequate to cover the variations and extra-grammaticality of users' input. In contrast, an $n$-gram language model derives its robustness by permitting all strings over an alphabet, albeit with different likelihoods. In an attempt to provide robustness to the grammar-based model, we created a corpus $\left(C_{\text {gram }}\right)$ of $k$ sentences by randomly sampling the set of paths of the grammar $\left(G_{g r a m}\right)^{6}$ and built a class-based $n$-gram language model $\left(\lambda_{\text {gram }}\right)$ using this corpus. Although this corpus does not represent the true distribution of sentences in the MATCH domain, we are able to derive some of the benefits of $n$-gram language modeling techniques. Similar approaches have been presented in Galescu, Ringger, and Allen (1998) and Wang and Acero (2003).

\subsection{Combining Grammar and Corpus}

A straightforward extension of the idea of sampling the grammar in order to create a corpus is to select those sentences out of the grammar which make the resulting corpus "similar" to the corpus collected in the pilot studies. In order to create this corpus $C_{\text {closer }}$ we choose the $k$ most likely sentences as determined by a language model $\left(\lambda_{\text {MATCH }}\right)$ built using the collected corpus. A mixture model $\left(\lambda_{\text {mix }}\right)$ with mixture weight $(\alpha)$ is built by interpolating the model trained on the corpus of extracted sentences $\left(\lambda_{\text {close }}\right)$ and the model trained on the collected corpus $\left(\lambda_{M A T C H}\right)$. This method is summarized in Equation (4), where $L(M)$ represents the language recognized by the multimodal grammar $(M)$.

$$
\begin{aligned}
C_{\text {close }}= & \left\{S_{1}, \ldots S_{k} \mid S_{i} \in L(M) \wedge \operatorname{Pr}_{\lambda_{\text {MATCH }}}\left(S_{i}\right)>\operatorname{Pr}_{\lambda_{\text {MATCH }}}\left(S_{i+1}\right)\right. \\
& \wedge \nexists j \operatorname{Pr}_{\lambda_{\text {MATCH }}}\left(S_{i}\right)>\operatorname{Pr}_{\lambda_{M A T C H}}\left(S_{j}\right)>\operatorname{Pr}_{\lambda_{M A T C H}}\left(S_{i+1}\right) \\
\lambda_{\text {mix }}= & \alpha * \lambda_{\text {close }}+(1-\alpha) * \lambda_{M A T C H}
\end{aligned}
$$

6 We can also randomly sample a sub-network without expanding the $k$ paths. 


\subsection{Class-Based Out-of-Domain Language Model}

An alternative to using in-domain corpora for building language models is to "migrate" a corpus of a different domain to our domain. The process of migrating a corpus involves suitably generalizing the corpus to remove information that is specific only to the other domain and instantiating the generalized corpus to our domain. Although there are a number of ways of generalizing the out-of-domain corpus, the generalization we have investigated involved identifying linguistic units, such as noun and verb chunks, in the out-of-domain corpus and treating them as classes. These classes are then instantiated to the corresponding linguistic units from the MATCH domain. The identification of the linguistic units in the out-of-domain corpus is done automatically using a supertagger (Bangalore and Joshi 1999). We use a corpus collected in the context of a software help-desk application as an example out-of-domain corpus. In cases where the out-of-domain corpus is closely related to the domain at hand, a more semantically driven generalization might be more suitable. Figure 29 illustrates the process of migrating data from one domain to another.

\subsection{Adapting the Switchboard Language Model}

We investigated the performance of a large-vocabulary conversational speech recognition system when applied to a specific domain such as MATCH. We used the Switchboard corpus $\left(C_{s w b d}\right)$ as an example of a large-vocabulary conversational speech corpus. We built a trigram model $\left(\lambda_{s w b d}\right)$ using the 5.4-million-word corpus and investigated the effect of adapting the Switchboard language model given $k$ in-domain untranscribed speech utterances $\left(\left\{O_{M}^{i}\right\}\right)$. The adaptation is done by first recognizing the in-domain speech utterances and then building a language model $\left(\lambda_{\text {adapt }}\right)$ from the corpus of recognized text $\left(C_{\text {adapt }}\right)$. This bootstrapping mechanism can be used to derive a domainspecific corpus and language model without any transcriptions. Similar techniques for

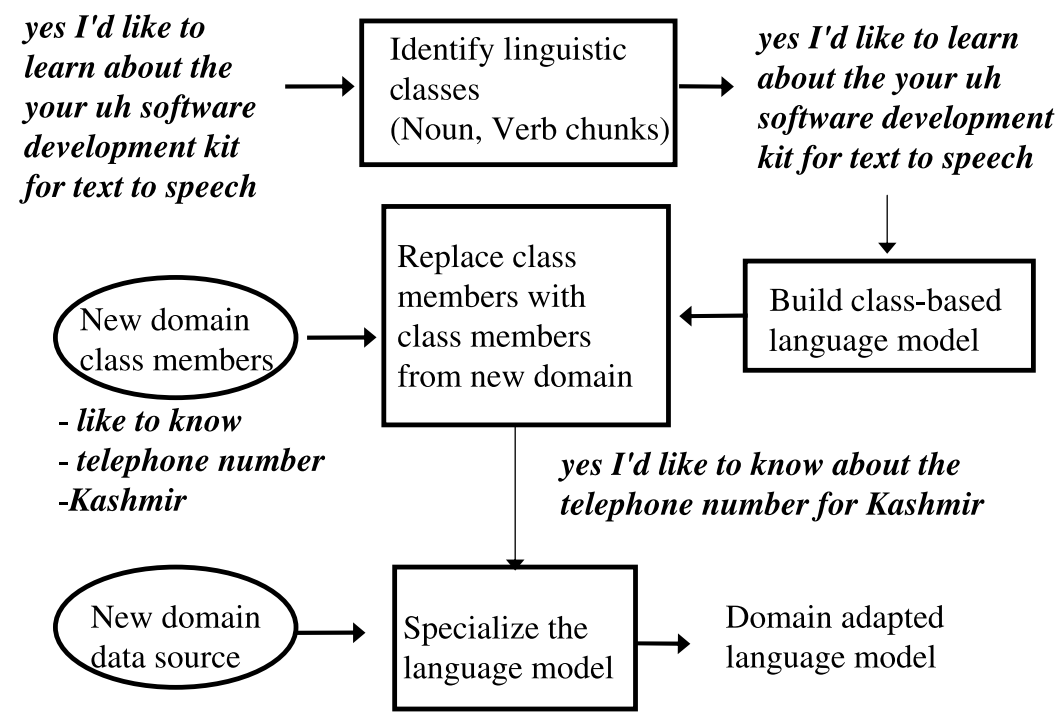

Figure 29

A method for migration of data from one domain to another domain. 
unsupervised language model adaptation are presented in Bacchiani and Roark (2003) and Souvignier and Kellner (1998).

$$
\begin{aligned}
C_{\text {adapt }} & =\left\{S_{1}, S_{2}, \ldots, S_{k}\right\} \\
S_{i} & =\underset{S}{\operatorname{argmax}} \pi_{2}\left(O_{M}^{i} \circ A \circ L \circ G_{s w b d}\right)(S)
\end{aligned}
$$

\subsection{Adapting a Wide-Coverage Grammar}

There have been a number of computational implementations of wide-coverage, domain-independent, syntactic grammars for English in various grammar formalisms (Flickinger, Copestake, and Sag 2000; XTAG 2001; Clark and Hockenmaier 2002). Here, we describe a method that exploits one such grammar implementation in the Lexicalized Tree-Adjoining Grammar (LTAG) formalism, for deriving domain-specific corpora. An LTAG consists of a set of elementary trees (supertags) (Bangalore and Joshi 1999) each associated with a lexical item (the head). Supertags encode predicate-argument relations of the head and the linear order of its arguments with respect to the head. In Figure 30, we show the supertag associated with the word show in an imperative sentence such as show the Empire State Building. A supertag can be represented as a finite-state machine with the head and its arguments as arc labels (Figure 31). The set of sentences generated by an LTAG can be obtained by combining supertags using substitution and adjunction operations. In related work (Rambow et al. 2002), it has been shown that for a restricted version of LTAG, the combinations of a set of supertags can be represented as an FSM. This FSM compactly encodes the set of sentences generated by an LTAG grammar. It is composed of two transducers, a lexical FST, and a syntactic FSM.

The lexical FST transduces input words to supertags. We assume that as input to the construction of the lexical machine we have a list of words with their parts-of-speech. Once we have determined for each word the set of supertags they should be associated

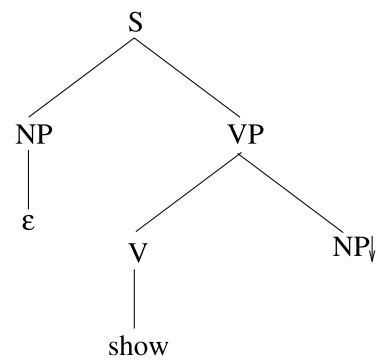

Figure 30

Supertag tree for the word show. The NP nodes permit substitution of all supertags with root node labeled NP.

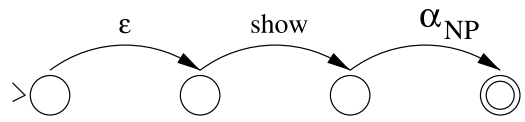

Figure 31

FSM for the word show. The $\alpha_{N P}$ arc permits replacement with FSMs representing NP. 
with, we create a disjunctive finite-state transducer (FST) for all words which transduces the words to their supertags.

For the syntactic FSM, we take the union of all the FSMs for each supertag which corresponds to an initial tree (i.e., a tree which need not be adjoined). We then perform a series of iterative replacements: In each iteration, we replace each arc labeled by a supertag by its lexicalized version of that supertag's FSM. Of course, in each iteration, there are many more replacements than in the previous iteration. Based on the syntactic complexity in our domain (such as number of modifiers, clausal embedding, and prepositional phrases), we use five rounds of iteration. The number of iterations restricts the syntactic complexity but not the length of the input. This construction is in many ways similar to constructions proposed for CFGs, in particular that of Nederhof (1997). One difference is that, because we start from TAG, recursion is already factored, and we need not find cycles in the rules of the grammar.

We derive a MATCH domain-specific corpus by constructing a lexicon consisting of pairings of words with their supertags that are relevant to this domain. We then compile the grammar to build an FSM of all sentences up to a given depth of recursion. We sample this FSM and build a language model as discussed in Section 5.3. Given untranscribed utterances from a specific domain, we can also adapt the language model as discussed in Section 5.6.

\subsection{Speech Recognition Experiments}

We describe a set of experiments to evaluate the performance of the language model in the MATCH multimodal system. We use word accuracy and string accuracy for evaluating ASR output. All results presented in this section are based on 10-fold crossvalidation experiments run on the 709 spoken and multimodal exchanges collected from the pilot study described in Section 2.2.

Table 4 presents the performance results for ASR word and sentence accuracy using language models trained on the collected in-domain corpus as well as on corpora derived using the different methods discussed in Sections 5.2-5.7. For the class-based models mentioned in the table, we defined different classes based on areas of interest (e.g., riverside park, turtle pond), points of interest (e.g., Ellis Island, United Nations Building), type of cuisine (e.g., Afghani, Indonesian), price categories (e.g., moderately priced, expensive), and neighborhoods (e.g., Upper East Side, Chinatown).

It is immediately apparent that the hand-crafted grammar as a language model performs poorly and a language model trained on the collected domain-specific corpus performs significantly better than models trained on derived data. However, it is encouraging to note that a model trained on a derived corpus (obtained from combining the migrated out-of-domain corpus and a corpus created by sampling the in-domain grammar) is within $10 \%$ word accuracy as compared to the model trained on the collected corpus. There are several other noteworthy observations from these experiments.

The performance of the language model trained on data sampled from the grammar is dramatically better as compared to the performance of the hand-crafted grammar. This technique provides a promising direction for authoring portable grammars that can be sampled subsequently to build robust language models when no in-domain corpora are available. Furthermore, combining grammar and in-domain data, as described in Section 5.4, outperforms all other models significantly.

For the experiment on the migration of an out-of-domain corpus, we used a corpus from a software help-desk application. Table 4 shows that the migration of data using 
Table 4

Performance results for ASR word and sentence accuracy using models trained on data derived from different methods of bootstrapping domain-specific data.

\begin{tabular}{|c|c|c|c|c|}
\hline & & Scenario & $\begin{array}{l}\text { ASR Word } \\
\text { Accuracy }\end{array}$ & $\begin{array}{l}\text { Sentence } \\
\text { Accuracy }\end{array}$ \\
\hline \multirow[t]{2}{*}{1} & \multirow[t]{2}{*}{ Grammar-based } & $\begin{array}{l}\text { Grammar as language model } \\
\text { (Section 5.2) }\end{array}$ & 41.6 & 38.0 \\
\hline & & $\begin{array}{l}\text { Class-based } n \text {-gram language } \\
\text { model (Section 5.3) }\end{array}$ & 60.6 & 42.9 \\
\hline 2 & In-domain Data & $\begin{array}{l}\text { Class-based } n \text {-gram model } \\
\text { (Section 5.1) }\end{array}$ & 73.8 & 57.1 \\
\hline 3 & $\begin{array}{l}\text { Grammar+ } \\
\text { In-domain Data }\end{array}$ & $\begin{array}{l}\text { Class-based } n \text {-gram model } \\
\text { (Section 5.4) }\end{array}$ & 75.0 & 59.5 \\
\hline \multirow[t]{4}{*}{4} & \multirow{4}{*}{$\begin{array}{l}\text { Out-of-domain } \\
\text { (Section 5.5) }\end{array}$} & $n$-gram model & 17.6 & 17.5 \\
\hline & & Class-based $n$-gram model & 58.4 & 38.8 \\
\hline & & $\begin{array}{l}\text { Class-based } n \text {-gram model } \\
\text { with Grammar-based } n \text {-gram }\end{array}$ & & \\
\hline & & Language Model & 64.0 & 45.4 \\
\hline \multirow[t]{2}{*}{5} & \multirow{2}{*}{$\begin{array}{l}\text { Switchboard } \\
\text { (Section 5.6) }\end{array}$} & $n$-gram model & 43.5 & 25.0 \\
\hline & & $\begin{array}{l}\text { Language model trained on } \\
\text { recognized in-domain data }\end{array}$ & 55.7 & 36.3 \\
\hline \multirow[t]{2}{*}{6} & \multirow{2}{*}{$\begin{array}{l}\text { Wide-coverage } \\
\text { Grammar } \\
\text { (Section 5.7) }\end{array}$} & $n$-gram model & 43.7 & 24.8 \\
\hline & & $\begin{array}{l}\text { Language model trained on } \\
\text { recognized in-domain data }\end{array}$ & 55.8 & 36.2 \\
\hline
\end{tabular}

linguistic units as described in Section 5.5 significantly outperforms a model trained only on the out-of-domain corpus. Also, combining the grammar sampled corpus with the migrated corpus provides further improvement.

The performance of the Switchboard model on the MATCH domain is presented in the fifth row of Table 4. We built a trigram model using a 5.4-million-word Switchboard corpus and investigated the effect of adapting the resulting language model on in-domain untranscribed speech utterances. The adaptation is done by first running the recognizer on the training partition of the in-domain speech utterances and then building a language model from the recognized text. We observe that although the performance of the Switchboard language model on the MATCH domain is poorer than the performance of a model obtained by migrating data from a related domain, the performance can be significantly improved using the adaptation technique.

The last row of Table 4 shows the results of using the MATCH specific lexicon to generate a corpus using a wide-coverage grammar, training a language model, and adapting the resulting model using in-domain untranscribed speech utterances as was done for the Switchboard model. The class-based trigram model was built using 500,000 randomly sampled paths from the network constructed by the procedure described in Section 5.7. It is interesting to note that the performance is very similar to the Switchboard model given that the wide-coverage grammar is not designed for conversational speech unlike models derived from Switchboard data. The data from the domain has some elements of conversational-style speech which the Switchboard model models well, but it also has syntactic constructions that are adequately modeled by the widecoverage grammar. 
In this section, we have presented a range of techniques to build language models for speech recognition which are applicable at different development phases of an application. Although the utility of in-domain data cannot be obviated, we have shown that there are ways to approximate this data with a combination of grammar and out-ofdomain data. These techniques are particularly useful in the initial phases of application development when there is very little in-domain data. The technique of authoring a domain-specific grammar that is sampled for $n$-gram model building presents a good trade-off between time-to-create and the robustness of the resulting language model. This method can be extended by incorporating suitably generalized out-of-domain data, in order to approximate the distribution of $n$-grams in the in-domain data. If time to develop is of utmost importance, we have shown that using a large out-of-domain corpus (Switchboard) or a wide-coverage domain-independent grammar can yield a reasonable language model.

\section{Robust Multimodal Understanding}

In Section 3, we showed how multimodal grammars can be compiled into finite-state transducers enabling effective processing of lattice input from speech and gesture recognition and mutual compensation for errors and ambiguities. However, like other approaches based on hand-crafted grammars, multimodal grammars can be brittle with respect to extra-grammatical, erroneous, and disfluent input. Also, the primary applications of multimodal interfaces include use in noisy mobile environments and use by inexperienced users (for whom they provide a more natural interaction); therefore it is critical that multimodal interfaces provide a high degree of robustness to unexpected or ill-formed inputs.

In the previous section, we presented methods for bootstrapping domain-specific corpora for the purpose of training robust language models used for speech recognition. These methods overcome the brittleness of a grammar-based language model. Although the corpus-driven language model might recognize a user's utterance correctly, the recognized utterance may not be assigned a semantic representation by the multimodal grammar if the utterance is not part of the grammar.

In this section, we address the issue of robustness in multimodal understanding. Robustness in multimodal understanding results from improving robustness to speech recognition and gesture recognition errors. Although the techniques in this section are presented as applying to the output of a speech recognizer, they are equally applicable to the output of a gesture recognizer. We chose to focus on robustness to speech recognition errors because the errors in a gesture recognizer are typically smaller than in a speech recognizer due to smaller vocabulary and lower perplexity.

There have been two main approaches to improving robustness of the understanding component in the spoken language understanding literature. First, a parsingbased approach attempts to recover partial parses from the parse chart when the input cannot be parsed in its entirety due to noise, in order to construct a (partial) semantic representation (Ward 1991; Dowding et al. 1993; Allen et al. 2001). Second, a classification-based approach, adopted from the Information Extraction literature, views the problem of understanding as extracting certain bits of information from the input. It attempts to classify the utterance and identifies substrings of the input as slotfiller values to construct a frame-like semantic representation. Both approaches have limitations. Although in the first approach the grammar can encode richer semantic representations, the method for combining the fragmented parses is quite ad hoc. In the 
second approach, the robustness is derived from training classifiers on annotated data; this data is very expensive to collect and annotate, and the semantic representation is fairly limited. There is some more recent work on using structured classification approaches to transduce sentences to logical forms (Papineni, Roukos, and Ward 1997; Thompson and Mooney 2003; Zettlemoyer and Collins 2005). However, it is not clear how to extend these approaches to apply to lattice input-an important requirement for multimodal processing.

\subsection{Evaluation Issue}

Before we present the methods for robust understanding, we discuss the issue of data partitions to evaluate these methods on. Due to the limited amount of data, we run cross-validation experiments in order to arrive at reliable performance estimates for these methods. However, we have a choice in terms of how the data is split into training and test partitions for the cross-validation runs. We could randomly split the data for an $n$-fold (for example, 10 -fold) cross-validation test. However, the data contain several repeated attempts by users performing the six scenarios. A random partitioning of these data would inevitably have the same multimodal utterances in training and test partitions. We believe that this would result in an overly optimistic estimate of the performance. In order to address this issue, we run 6-fold cross-validation experiments by using five scenarios as the training set and the sixth scenario as the test set. This way of partitioning the data overly handicaps data-driven methods because the distribution of data in the training and test partitions for each cross-validation run would be significantly different. In the experiment results for each method, we present 10-fold and 6-fold cross-validation results where appropriate in order to demonstrate the strengths and limitations of each method. For all the experiments in this section, we used a datadriven language model for ASR. The word accuracy of the ASR is $73.8 \%$, averaged over all scenarios and all speakers.

\subsection{Classification-Based Approach}

In this approach we view robust multimodal understanding as a sequence of classification problems in order to determine the predicate and arguments of an utterance. The set of predicates are the same set of predicates used in the meaning representation. The meaning representation shown in Figure 28 consists of a predicate (the command attribute) and a sequence of one or more argument attributes which are the parameters for the successful interpretation of the user's intent. For example, in Figure 28, $\mathrm{cmd}$ : info is the predicate and type:phone object:selection are the arguments to the predicate.

We determine the predicate $\left(c^{*}\right)$ for a $N$ token multimodal utterance $\left(S_{1}^{N}\right)$ by searching for the predicate $(c)$ that maximizes the posterior probability as shown in Equation (6).

$$
c^{*}=\underset{c}{\operatorname{argmax}} P\left(c \mid S_{1}^{N}\right)
$$

We view the problem of identifying and extracting arguments from a multimodal input as a problem of associating each token of the input with a specific tag that encodes 
the label of the argument and the span of the argument. These tags are drawn from a tagset which is constructed by extending each argument label by three additional symbols $I, O, B$, following Ramshaw and Marcus (1995). These symbols correspond to cases when a token is inside $(I)$ an argument span, outside $(O)$ an argument span, or at the boundary of two argument spans (B) (See Table 5).

Given this encoding, the problem of extracting the arguments amounts to a search for the most likely sequence of tags $\left(T^{*}\right)$ given the input multimodal utterance $S_{1}^{N}$ as shown in Equation (7). We approximate the posterior probability $P\left(T \mid S_{1}^{N}\right)$ using independence assumptions to include the lexical context in an $n$-word window and the preceding two tag labels, as shown in Equation (8).

$$
\begin{aligned}
T^{*} & =\underset{T}{\operatorname{argmax}} P\left(T \mid S_{1}^{N}\right) \\
& \approx \underset{T}{\operatorname{argmax}} \prod_{i} P\left(t_{i} \mid S_{i-n}^{i}, S_{i+1}^{i+n+1}, t_{i-1}, t_{i-2}\right)
\end{aligned}
$$

Owing to the large set of features that are used for predicate identification and argument extraction, which typically result in sparseness problems for generative models, we estimate the probabilities using a classification model. In particular, we use the Adaboost classifier (Schapire 1999) wherein a highly accurate classifier is built by combining many "weak" or "simple" base classifiers $f_{i}$, each of which may only be moderately accurate. The selection of the weak classifiers proceeds iteratively, picking the weak classifier that correctly classifies the examples that are misclassified by the previously-selected weak classifiers. Each weak classifier is associated with a weight $\left(w_{i}\right)$ that reflects its contribution towards minimizing the classification error. The posterior probability of $P(c \mid x)$ is computed as in Equation 9. For our experiments, we use simple $n$-grams of the multimodal utterance to be classified as weak classifiers.

$$
P(c \mid x)=\frac{1}{\left(1+e^{-2 * \sum_{i} w_{i} * f_{i}(x)}\right)}
$$

For the experiments presented subsequently we use the data collected from the domain to train the classifiers. However, the data could be derived from an in-domain grammar using techniques similar to those presented in Section 5.

Table 5

The $\{\mathrm{I}, \mathrm{O}, \mathrm{B}\}$ encoding for argument extraction.

User cheap thai upper west side

Utterance

Argument $<$ price $>$ cheap $</$ price $><$ cuisine $>$

Annotation thai $</$ cuisine $><$ place $>$ upper west side $</$ place $>$

IOB

Encoding

cheap_price $<$ B $>$ thai_cuisine $<$ B $>$ upper_place $<$ I $>$ west_place $<$ I $>$ side_place $<\mathrm{I}>$ 
6.2.1 Experiments and Results. We used a total of 10 predicates such as help, assert, inforequest, and 20 argument types such as cuisine, price, location for our experiments. These were derived from our meaning representation language. We used unigrams, bigrams, and trigrams appearing in the multimodal utterance as weak classifiers for the purpose of predicate classification. In order to predict the tag of a word for argument extraction, we used the left and right trigram context and the tags for the preceding two tokens as weak classifiers. The results are presented in Table 6 . We present the concept sentence accuracy and the predicate and argument string accuracy of the grammar-based understanding model and the classification-based understanding model. The corresponding accuracy results on the 10-fold cross-validation experiments are shown in parentheses. As can be seen, the grammar-based model significantly outperforms the classification-based approach on the 6-fold cross-validation experiments and the classification-based approach outperforms the grammar-based approach on the 10-fold cross-validation experiments. This is to be expected since the classificationbased approach needs to generalize significantly from the training set to the test set, and these have different distributions of predicates and arguments in the 6-fold crossvalidation experiments.

A significant shortcoming of the classification approach is that it does not exploit the semantic grammar from the MATCH domain to constrain the possible choices from the classifier. Also, the classifier is trained using the data that is collected in this domain. However, the grammar is a rich source of distribution-free data. It is conceivable to sample the grammar in order to increase the training examples for the classifier, in the same spirit as was done for building a language model using the grammar (Section 5.3). Furthermore, knowledge encoded in the grammar and data can be combined by techniques presented in Schapire et al. (2002) to improve classifier performance.

Another limitation of this approach is that it is unclear how to extend it to apply to speech and gesture lattices. As shown in earlier sections, multimodal understanding receives ambiguous speech and gesture inputs encoded as lattices. Mutual disambiguation between these two modalities needs to be exploited. Although the classification approach can be extended to apply to $n$-best lists of speech and gesture inputs, we prefer an approach that can apply to lattice inputs directly.

\subsection{Noisy Channel Model for Error Correction}

In order to address the limitations of the classification-based approach, we explore an alternate method for robust multimodal understanding. We translate the user's input to a string that can be assigned a meaning representation by the grammar. We can

Table 6

Concept accuracy results from classification-based model using data-driven language model for ASR. (Numbers are percent for 6-fold cross validation by scenario. Corresponding percent for 10 -fold cross validation are given in parentheses.)

\begin{tabular}{lccc}
\hline Model & $\begin{array}{c}\text { Concept } \\
\text { Sentence } \\
\text { Accuracy \% }\end{array}$ & $\begin{array}{c}\text { Predicate } \\
\text { Sentence } \\
\text { Accuracy \% }\end{array}$ & $\begin{array}{c}\text { Argument } \\
\text { Sentence } \\
\text { Accuracy \% }\end{array}$ \\
\hline Grammar-based & $38.9(41.5)$ & $40.3(43.1)$ & $40.7(43.2)$ \\
Classification-based & $34.0(58.3)$ & $71.4(85.5)$ & $32.8(61.4)$
\end{tabular}


apply this technique on a user's gesture input as well in order to compensate for gesture recognition errors. We couch the problem of error correction in the noisy channel modeling framework. In this regard, we follow Ringger and Allen (1996) and Ristad and Yianilos (1998); however, we encode the error correction model as a weighted FST so we can directly edit speech/gesture input lattices. As mentioned earlier, we rely on integrating speech and gesture lattices to avoid premature pruning of admissible solutions for robust multimodal understanding. Furthermore, unlike Ringger and Allen, the language grammar from our application filters out edited strings that cannot be assigned an interpretation by the multimodal grammar. Also, whereas in Ringger and Allen the goal is to translate to the reference string and improve recognition accuracy, in our approach the goal is to translate the input in order to assign the reference meaning and improve concept accuracy.

We let $S_{g}$ be the string that can be assigned a meaning representation by the grammar and $S_{u}$ be the user's input utterance. If we consider $S_{u}$ to be the noisy version of the $S_{g}$, we view the decoding task as a search for the string $S_{g}^{*}$ as shown in Equation (10). Note we formulate this as a joint probability maximization as in Equation (11). Equation (12) expands the sequence probability by the chain rule where $S_{u}^{i}$ and $S_{g}^{i}$ are the $i^{\text {th }}$ tokens from $S_{u}$ and $S_{g}$ respectively. We use a Markov approximation (limiting the dependence on the history to the past two time steps: trigram assumption for our purposes) to compute the joint probability $P\left(S_{u}, S_{g}\right)$, shown in Equation (13).

$$
\begin{aligned}
& S_{g}^{*}=\underset{S_{g}}{\operatorname{argmax}} P\left(S_{g} \mid S_{u}\right) \\
& =\underset{S_{g}}{\operatorname{argmax}} P\left(S_{g}, S_{u}\right) \\
& =\underset{S_{g}}{\operatorname{argmax}} P\left(S_{u}^{0}, S_{g}^{0}\right) * P\left(S_{u}^{1}, S_{g}^{1} \mid S_{u}^{0}, S_{g}^{0}\right) \ldots * P\left(S_{u}^{n}, S_{g}^{n} \mid S_{u}^{0}, S_{g}^{0}, \ldots, S_{u}^{n-1}, S_{g}^{n-1}\right) \\
& S_{g}^{*}=\underset{S_{g}}{\operatorname{argmax}} \prod P\left(S_{u}^{i}, S_{g}^{i} \mid S_{u}^{i-1}, S_{u}^{i-2}, S_{g}^{i-1}, S_{g}^{i-2}\right)
\end{aligned}
$$

where $S_{u}=S_{u}^{1} S_{u}^{2} \ldots S_{u}^{n}$ and $S_{g}=S_{g}^{1} S_{g}^{2} \ldots S_{g}^{m}$.

In order to compute the joint probability, we need to construct an alignment between tokens $\left(S_{u}^{i}, S_{g}^{i}\right)$. We use the Viterbi alignment provided by the GIZA++ toolkit (Och and Ney 2003) for this purpose. We convert the Viterbi alignment into a bilanguage representation that pairs words of the string $S_{u}$ with words of $S_{g}$. A few examples of bilanguage strings are shown in Figure 32. We compute the joint $n$-gram model using a language modeling toolkit (Goffin et al. 2005). Equation (13) thus allows us to edit a user's utterance to a string that can be interpreted by the grammar.

1. show:show me:me the: $\epsilon$ map: $\epsilon$ of: $\epsilon$ midtown:midtown

2. no: $\epsilon$ find:find me:me french:french restaurants:around downtown:downtown

3. I: $\epsilon$ need: $\epsilon$ subway:subway directions:directions

\section{Figure 32}

A few examples of bilanguage strings. 
6.3.1 Deriving a Translation Corpus. Because our multimodal grammar is implemented as a finite-state transducer it is fully reversible and can be used not just to provide a meaning for input strings but can also be run in reverse to determine possible input strings for a given meaning. Our multimodal corpus was annotated for meaning using the multimodal annotation tools described in Section 2.2. In order to train the translation model we built a corpus that pairs the reference speech string for each utterance in the training data with a target string. The target string is derived in two steps. First, the multimodal grammar is run in reverse on the reference meaning yielding a lattice of possible input strings. Second, the closest string (as defined by Levenshtein editdistance [Levenshtein 1966]) in the lattice to the reference speech string is selected as the target string.

6.3.2 FST-Based Decoder. In order to facilitate editing of ASR lattices, we represent the $n$-gram translation model as a weighted finite-state transducer (Bangalore and Riccardi 2002). We first represent the joint $n$-gram model as a finite-state acceptor (Allauzen et al. 2004). We then interpret the symbols on each arc of the acceptor as having two components-a word from the user's utterance (input) and a word from the edited string (output). This transformation makes a transducer out of an acceptor. In doing this, we can directly compose the editing model $\left(\lambda_{M T}\right)$ with ASR lattices $\left(\lambda_{S}\right)$ to produce a weighted lattice of edited strings. We further constrain the set of edited strings to those that are interpretable by the grammar. We achieve this by composing with the language finite-state acceptor derived from the multimodal grammar $\left(\lambda_{G}\right)$ and searching for the best edited string, as shown in Equation (14).

$$
S_{M T}^{*}=\underset{S}{\operatorname{argmax}} \lambda_{S} \circ \lambda_{M T} \circ \lambda_{G}
$$

If we were to apply this approach to input gesture lattices, then the translation model would be built from pairings of the gesture recognition output and the corresponding gesture string that would be interpretable by the grammar. Typical errors in gesture input could include misrecognition of a spurious gesture that ought to have been treated as noise (caused, for example, by improper detection of a pen-down event) and non-recognition of pertinent gestures due to early end-pointing of ink input.

Figure 33 shows two examples. In the first example, the unimodal speech utterance was edited by the model to produce a string that was correctly interpreted by the multimodal grammar. In the second example, the speech and gesture integration failed and resulted in an empty meaning representation. However, after the edit on the speech string, the multimodal utterance was correctly interpreted.

6.3.3 Experiments and Results. Table 7 summarizes the results of the translation model (TM) and compares its accuracy to a grammar-based model. We provide concept accuracy and predicate and argument string accuracy of the translation models applied to one-best and lattice ASR input. We also provide concept accuracy results on the lattice of edited strings resulting from applying the translation models to the user's input. As can be seen from the table, the translation models outperform the grammarbased models significantly in all cases. It is also interesting to note that there is some improvement in concept accuracy using an ASR lattice over a one-best ASR output with one-best translation output. However, the improvement is much more significant using a lattice output from the translation model, suggesting that delaying selection of the edited string until the grammar/domain constraints are applied is paying off. 
Speech Input: I'm trying to find african restaurants that are located west of midtown

Gesture Input: <empty>

Edited Output: find african around west midtown

Meaning Before Edit: <empty>

Meaning After Edit: cmd:info type:restaurant cuisine:african location:west_midtown

Input: I'd like directions subway directions from the metropolitan museum of art to this place

Gesture Input: $\mathrm{G}$ area sel 1 rest $\operatorname{SEM}([\mathrm{id} 1])$

Edited Output: subway directions from the metropolitan museum of art to this place

Meaning Before Edit: <empty>

Meaning After Edit: cmd:route destination: [id1]

source: metropolitan_museum_of_art

Figure 33

Sample inputs and the edited outputs from the translation model.

One of the limitations of the translation model is that the edit operations that are learned are entirely driven by the parallel data. However, when the data are limited, as is the case here, the edit operations learned are also restricted. We would like to incorporate domain-specific edit operations in addition to the ones that are reflected in the data. In the next section, we explore this approach.

\subsection{Edit-Based Approach}

In this section, we extend the approach of translating the "noisy" version of the user's input to the "clean" input to incorporate arbitrary editing operations. We encode the possible edits on the input string as an edit FST with substitution, insertion, deletion, and identity arcs. These operations could be either word-based or phone-based and are associated with a cost. This allows us to incorporate, by hand, a range of edits that may not have been observed in the data used in the noisy-channel-based errorcorrection model. The edit transducer coerces the set of strings $(\mathcal{S})$ encoded in the lattice resulting from ASR $\left(\lambda_{\mathcal{S}}\right)$ to the closest strings in the grammar that can be assigned an interpretation. We are interested in the string with the least-cost sequence of edits

\section{Table 7}

Concept sentence accuracy of grammar-based and translation-based models with data-driven language model for ASR. (Numbers are percent for 6-fold cross validation by scenario. Corresponding percent for 10 -fold cross validation are given in parentheses.)

\begin{tabular}{lccc}
\hline Model & $\begin{array}{c}\text { Concept } \\
\text { Sentence } \\
\text { Accuracy (\%) }\end{array}$ & $\begin{array}{c}\text { Predicate } \\
\text { Sentence } \\
\text { Accuracy (\%) }\end{array}$ & $\begin{array}{c}\text { Argument } \\
\text { Sentence } \\
\text { Accuracy (\%) }\end{array}$ \\
\hline Grammar-based & $38.9(41.5)$ & $40.3(43.1)$ & $40.7(43.2)$ \\
ASR 1-best/1-best TM & $46.1(61.6)$ & $68.0(70.5)$ & $47.0(62.6)$ \\
ASR 1-best/Lattice TM & $50.3(61.6)$ & $70.5(70.5)$ & $51.2(62.6)$ \\
ASR Lattice/1-best TM & $46.7(61.9)$ & $70.8(69.9)$ & $47.1(63.3)$ \\
ASR Lattice/Lattice TM & $54.2(60.8)$ & $81.9(67.2)$ & $54.5(61.6)$
\end{tabular}




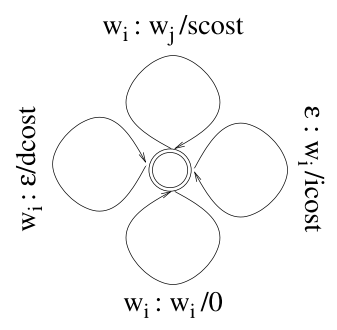

Figure 34

Basic edit machine.

(argmin) that can be assigned an interpretation by the grammar. ${ }^{7}$ This can be achieved by composition (o) of transducers followed by a search for the least-cost path through a weighted transducer as shown in Equation (15).

$$
s^{*}=\underset{s \in \mathcal{S}}{\operatorname{argmin}} \lambda_{\mathcal{S}} \circ \lambda_{\text {edit }} \circ \lambda_{g}
$$

We first describe the machine introduced in Bangalore and Johnston (2004) (Basic edit) then go on to describe a smaller edit machine with higher performance (4-edit) and an edit machine which incorporates additional heuristics (Smart edit).

6.4.1 Basic Edit. The edit machine described in Bangalore and Johnston (2004) is essentially a finite-state implementation of the algorithm to compute the Levenshtein distance. It allows for unlimited insertion, deletion, and substitution of any word for another (Figure 34). The costs of insertion, deletion, and substitution are set as equal, except for members of classes such as price (expensive), cuisine (turkish), and so on, which are assigned a higher cost for deletion and substitution.

6.4.2 Limiting the Number of Edits. Basic edit is effective in increasing the number of strings that are assigned an interpretation (Bangalore and Johnston 2004) but is quite large (15Mb, 1 state, 978,120 arcs) and adds an unacceptable amount of latency (5 seconds on average) in processing one-best input and is computationally prohibitive to use on lattices. In order to overcome these performance limitations, we experimented with revising the topology of the edit machine so that it allows only a limited number of edit operations (e.g., at most four edits) and removed the substitution arcs, because they give rise to $O\left(\left|\sum\right|^{2}\right)$ arcs, where $\sum$ is the vocabulary. Substitution is still possible but requires one delete and one insert. For the same grammar, the resulting edit machine is about $300 \mathrm{~Kb}$ with 4 states and 16,796 arcs. The topology of the 4-edit machine is shown in Figure 35. In addition to 4-edit, we also investigated 6-edit and 8-edit machines whose results we report in the subsequent sections.

There is a significant savings in bounding the number of edits on the number of paths in the resulting lattice. After composing with the basic edit machine, the lattice would contain $O\left(n *\left|\sum\right|\right)$ arcs where $n$ is the length of the input being edited. For the bounded $k$-edit machines this reduces to $O(k *|\Sigma|)$ arcs and $O\left(|\Sigma|^{k}\right)$ paths for a constant $k$.

7 Note that the closest string according to the edit metric may not be the closest string in meaning. 


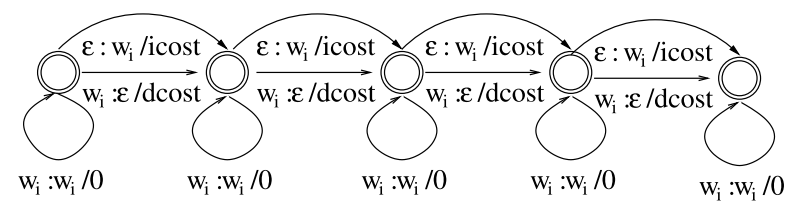

Figure 35

4-edit machine.

6.4.3 Smart Edit. We incorporate a number of additional heuristics and refinements to tune the 4-edit machine based on the underlying application database.

i. Deletion of SLM-only words We add arcs to the edit transducer to allow for free deletion of words in the SLM training data which are not found in the grammar: for example, listings in thai restaurant listings in midtown $\rightarrow$ thai restaurant in midtown.

ii. Deletion of doubled words A common error observed in SLM output was doubling of monosyllabic words: for example, subway to the cloisters recognized as subway to to the cloisters. We add arcs to the edit machine to allow for free deletion of any short word when preceded by the same word.

iii. Extended variable weighting of words Insertion and deletion costs were further subdivided from two to three classes: a low cost for "dispensable" words, (e.g., please, would, looking, a, the), a high cost for special words (slot fillers, e.g., chinese, cheap, downtown), and a medium cost for all other words, (e.g., restaurant, find).

iv. Auto completion of place names It is unlikely that grammar authors will include all of the different ways to refer to named entities such as place names. For example, if the grammar includes metropolitan museum of art the user may just say metropolitan museum. These changes can involve significant numbers of edits. A capability was added to the edit machine to complete partial specifications of place names in a single edit. This involves a closed world assumption over the set of place names. For example, if the only metropolitan museum in the database is the metropolitan museum of art we assume that we can insert of art after metropolitan museum. The algorithm for construction of these auto-completion edits enumerates all possible substrings (both contiguous and non-contiguous) for place names. For each of these it checks to see if the substring is found in more than one semantically distinct member of the set. If not, an edit sequence is added to the edit machine which freely inserts the words needed to complete the place name. Figure 36 illustrates one of the edit transductions that is added for the place name metropolitan museum of art. The algorithm which generates the autocomplete edits also generates new strings to add to the place name class for the SLM (expanded class). In order to limit over-application of the completion mechanism, substrings starting in prepositions (of art $\rightarrow$ metropolitan museum of art) or involving deletion of parts of abbreviations are not considered for edits ( $b$ c building $\rightarrow n b c$ building).

Note that the application-specific structure and weighting of Smart edit (iii, iv) can be derived automatically: We use the place-name list for auto completion of place names and use the domain entities, as determined by which words correspond to fields in the underlying application database, to assign variable costs to different entities.

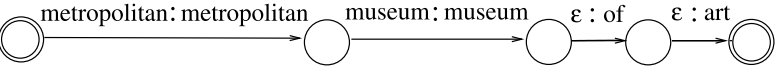

Figure 36

Auto-completion edits. 
Table 8

Concept accuracy for different edit models on 6-fold cross-validation experiments using a data-driven language model for ASR.

\begin{tabular}{lccc}
\hline Model & $\begin{array}{c}\text { Concept } \\
\text { Sentence } \\
\text { Accuracy (\%) }\end{array}$ & $\begin{array}{c}\text { Predicate } \\
\text { Sentence } \\
\text { Accuracy (\%) }\end{array}$ & $\begin{array}{c}\text { Argument } \\
\text { Sentence } \\
\text { Accuracy (\%) }\end{array}$ \\
\hline
\end{tabular}

\begin{tabular}{llll}
\hline $\begin{array}{l}\text { Grammar-based } \\
\text { (No edits) }\end{array}$ & 38.9 & 40.3 & 40.7 \\
Basic edit & 51.5 & 63.1 & 52.6 \\
4-edit & 53.0 & 62.6 & 53.9 \\
6-edit & 58.2 & 74.7 & 59.0 \\
8-edit & 57.8 & 75.7 & 58.6 \\
Smart 4-edit & 60.2 & 69.9 & 60.9 \\
Smart 6-edit & 60.2 & 73.7 & 61.3 \\
Smart 8-edit & 60.9 & 76.0 & 61.9 \\
Smart edit (exp) & 59.7 & 70.8 & 60.5 \\
Smart edit (exp, lattice) & 62.0 & 73.1 & 63.0 \\
Smart edit (lattice) & 63.2 & 73.7 & 64.0
\end{tabular}

6.4.4 Experiments and Results. We summarize the concept accuracy results from the 6-fold cross-validation experiments using the different edit machines previously discussed. We also repeat the concept accuracy results from the grammar-based model with no edits for a point of comparison. When compared to the baseline of $38.9 \%$ concept accuracy without edits (No edits), Basic edit gave a relative improvement of $32 \%$, yielding 51.5\% concept accuracy (Table 8 ). Interestingly, by limiting the number of edit operations as in 4-edit, we improved the concept accuracy (53\%) compared to Basic edit. The reason for this improvement is that for certain input utterances, the Basic edit model creates a very large edited lattice and the composition with the grammar fails due to memory restrictions. ${ }^{8}$ We also show improvement in concept accuracy by increasing the number of allowable edit operations (up to 8-edit). The concept accuracy improves with increasing number of edits but with diminishing relative improvements.

The heuristics in Smart edit clearly improve on the concept accuracy of the basic edit models with a relative improvement of $55 \%$ over the baseline. Smart edit (exp) shows the concept accuracy of Smart edit running on input from an ASR model with the expanded classes required for auto completion of place names. Inspection of individual partitions showed that, while the expanded classes did allow for the correction of errors on place names, the added perplexity in the ASR model from expanding classes resulted in errors elsewhere and an overall drop in concept accuracy of $0.5 \%$ compared to Smart edit without expanded classes. Using ASR lattices as input to the edit models further improved the accuracy to the best concept sentence accuracy score of $63.2 \%$, a relative improvement of $62.5 \%$ over the no-edit model. Lattice input also improved the performance of Smart edit with the expanded classes from $59.7 \%$ to $62 \%$.

To summarize, in Table 9 we tabulate the concept accuracy results from the best performing configuration of each of the robust understanding methods discussed in this section. It is clear that the techniques such as translation-based edit and Smart edit that can exploit the domain-specific grammar improve significantly over the classification-based

8 However, the concept accuracy for the $70 \%$ of utterances which are assigned a meaning using the basic edit model was about $73 \%$. 
Table 9

Summary of concept accuracy results from the different robust understanding techniques using data-driven language models for ASR.

\begin{tabular}{lccc}
\hline Model & $\begin{array}{c}\text { Concept } \\
\text { Sentence } \\
\text { Accuracy (\%) }\end{array}$ & $\begin{array}{c}\text { Predicate } \\
\text { Sentence } \\
\text { Accuracy (\%) }\end{array}$ & $\begin{array}{c}\text { Argument } \\
\text { Sentence } \\
\text { Accuracy (\%) }\end{array}$ \\
\hline $\begin{array}{l}\text { Grammar-based } \\
\text { (No edits) }\end{array}$ & $38.9(41.5)$ & $40.3(43.1)$ & $40.7(43.2)$ \\
Classification-based & $34.0(58.3)$ & $71.4(85.5)$ & $32.8(61.4)$ \\
Translation-based edit & $54.2(60.8)$ & $81.9(67.2)$ & $54.5(61.6)$ \\
Smart edit & $63.2(68.4)$ & $73.7(73.8)$ & $64.0(69.4)$
\end{tabular}

approach. Furthermore, the heuristics encoded in the smart edit technique that exploit the domain constraints outperform the translation-based edit technique that is entirely data-dependent.

We also show the results from the 10-fold cross-validation experiments in the table. As can be seen there is a significant improvement in concept accuracy for datadriven techniques (classification and translation-based edit) compared to the 6-fold cross-validation experiments. This is to be expected because the distributions estimated from the training set fit the test data better in the 10-fold experiments as against 6 -fold experiments.

Based on the results we have presented in this section, it would be pragmatic to rapidly build a hand-crafted grammar-based conversational system that can be made robust using stochastic language modeling techniques and edit-based understanding techniques. Once the system is deployed and data collected, then a judicious balance of data-driven and grammar-based techniques would maximize the performance of the system.

\section{Robust Gesture Processing}

Gesture recognition has a lower error rate than speech recognition in this application. Even so, gesture misrecognitions and incompleteness of the multimodal grammar in specifying speech and gesture combinations contribute to the number of utterances not being assigned a meaning. We address the issue of robustness to gesture errors in this section.

We adopted the edit-based technique used on speech utterances to improve robustness of multimodal understanding. However, unlike a speech utterance, a gesture string has a structured representation. The gesture string is represented as a sequence of attribute-values (e.g., gesture type takes values from \{area, line, point, handwriting $\}$ ) and editing a gesture representation implies allowing for replacements within the value set. We adopted a simple approach that allows for substitution and deletion of values for each attribute, in addition to the deletion of any gesture. We did not allow for insertions of gestures as it is not clear what specific content should be assigned to an inserted gesture. One of the problems is that if you have, for example, a selection of two items and you want to increase it to three selected items, it is not clear a priori which entity to add as the third item. We encoded the edit operations for gesture editing as a finite-state transducer just as we did for editing speech utterances. Figure 37 illustrates the gesture edit transducer with delc representing the delection cost and substc the substitution cost. This method of manipulating the gesture recognition lattice is similar to gesture 


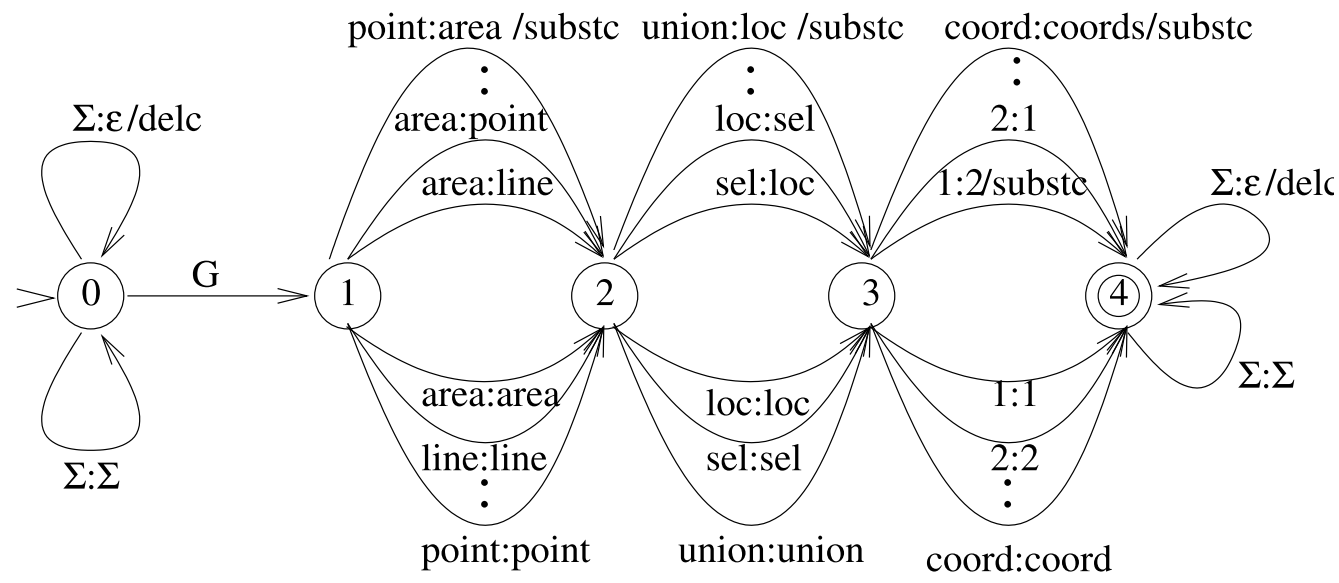

Figure 37

A finite-state transducer for editing gestures.

aggregation, introduced in Section 3.4. In contrast to substitution and deletion of gestures, gesture aggregation involves insertion of new gestures into the lattice; however, each introduced gesture has a well-defined meaning based on the combination of values of the gestures being aggregated.

We evaluated the effectiveness of the gesture edit machine on the MATCH data set. The data consisted of 174 multimodal utterances that were covered by the grammar. We used the transcribed speech utterance and the gesture lattice from the gesture recognizer as inputs to the multimodal integration and understanding system. For $55.4 \%$ of the utterances, we obtained the identical attribute-value meaning representation as the human-transcribed meaning representation.

Applying the gesture edit transducer on the gesture recognition lattices, and then integrating the result with the transcribed speech utterance produced a significant improvement in the accuracy of the attribute-value meaning representation. For $68.9 \%$ of the utterances, we obtained the identical attribute-value meaning representation as the human-transcribed meaning representation, a $22.5 \%$ absolute improvement in the robustness of the system that can be directly attributed to robustness in gesture integration and understanding. In future work, we would like to explore learning from data how to balance gesture editing and speech editing based on the relative reliabilities of the two modalities.

\section{Conclusion}

We view the contributions of the research presented in this article from two perspectives. First, we have shown how the finite-state approach to multimodal language processing (Johnston and Bangalore 2005) can be extended to support applications with complex pen input and how the approach can be made robust through coupling with a stochastic speech recognition model using translation techniques or finite-state edit machines. We have investigated the options available for bootstrapping domainspecific corpora for language models by exploiting domain-specific and wide-coverage grammars, linguistic generalization of out-of-domain data, and adapting domainindependent corpora. We have shown that such techniques can closely approximate the accuracy of speech recognizers trained on domain-specific corpora. For robust 
multimodal understanding we have presented and comparatively evaluated three different techniques based on discriminative classification, statistical translation, and edit machines. We have investigated the strengths and limitations of these approaches in terms of their ability to process lattice input, their ability to exploit constraints from a domain-specific grammar, and their ability to utilize domain knowledge from the underlying application database. The best performing multimodal understanding system, using a stochastic ASR model coupled with the smart 4-edit transducer on lattice input, is significantly more robust than the grammar-based system, achieving $68.4 \%$ concept sentence accuracy (10-fold) on data collected from novice first time users of a multimodal conversational system. This is a substantial $35 \%$ relative improvement in performance compared to $50.7 \%$ concept sentence accuracy (10-fold) using the grammar-based language and multimodal understanding models without edits. In our exploration of applying edit techniques to the gesture lattices we saw a $22.5 \%$ absolute improvement in robustness.

The second perspective on the work views it as an investigation of a range of techniques that balance the robustness provided by data-driven techniques and the flexibility provided by grammar-based approaches. In the past four decades of speech and natural language processing, both data-driven approaches and rule-based approaches have been prominent at different periods in time. Moderate-sized rule-based spoken language models for recognition and understanding are easy to develop and provide the ability to rapidly prototype conversational applications. However, scalability of such systems is a bottleneck due to the heavy cost of authoring and maintenance of rule sets and inevitable brittleness due to lack of coverage. In contrast, data-driven approaches are robust and provide a simple process of developing applications given availability of data from the application domain. However, this reliance on domainspecific data is also one of the significant bottlenecks of data-driven approaches. Development of conversational systems using data-driven approaches cannot proceed until data pertaining to the application domain is available. The collection and annotation of such data is extremely time-consuming and tedious, which is aggravated by the presence of multiple modalities in the user's input, as in our case. Also, extending an existing application to support an additional feature requires adding additional data sets with that feature. We have shown how a balanced approach where statistical language models are coupled with grammar-based understanding using edit machines can be highly effective in a multimodal conversational system. It is important to note that these techniques are equally applicable for speech-only conversational systems as well.

Given that the combination of stochastic recognition models with grammar-based understanding models provides robust performance, the question which remains is, after the initial bootstrapping phase, as more data becomes available, should this grammar-based approach be replaced with a data-driven understanding component? There are a number of advantages to the hybrid approach we have proposed which extend beyond the initial deployment of an application.

1. The expressiveness of the multimodal grammar allows us to specify any compositional relationships and meaning that we want. The range of meanings and their relationship to the input string can be arbitrarily simple or complex.

2. The multimodal grammar provides an alignment between speech and gesture input and enables multimodal integration of content from different modes. 
3. With the grammar-based approach it is straightforward to quickly add support for new commands to the grammar or change the representation of existing commands. The only retraining that is needed is for the ASR model, and data for the ASR model can either be migrated from another related domain or derived through grammar sampling.

4. Most importantly, this approach has the significant advantage that it does not require annotation of speech data with meaning representations and alignment of the meaning representations with word strings. This can be complex and expensive, involving a detailed labeling guide and instructions for annotators. In contrast in this approach, if data is used, all that is needed is transcription of the audio, a far more straightforward annotation task. If no data is used then grammar sampling can be used instead and no annotation of data is needed whatsoever.

5. Although data-driven approaches to understanding are commonplace in research, rule-based techniques continue to dominate in much of the industry (Pieraccini 2004). See, for example, the W3C SRGS standard (www.w3.org/TR/speech-grammar/).

\section{Acknowledgments}

We dedicate this article to the memory of Candy Kamm whose continued support for multimodal research made this work possible. We thank Patrick Ehlen, Helen Hastie, Preetam Maloor, Amanda Stent, Gunaranjan Vasireddy, Marilyn Walker, and Steve Whittaker for their contributions to the MATCH system. We also thank Richard Cox and Mazin Gilbert for their ongoing support of multimodal research at AT\&T Labs-Research. We would also like to thank the anonymous reviewers for their many helpful comments and suggestions for revision.

\section{References}

Abney, S. P. 1991. Parsing by chunks. In R. Berwick, S. Abney, and C. Tenny, editors, Principle-Based Parsing. IEEE, Los Alamitos, CA, pages 257-278.

Ades, A. E. and M. Steedman. 1982. On the order of words. Linquistics and Philosophu, 4:517-558.

Alexandersson, J. and T. Becker. 2001. Overlay as the basic operation for discourse processing in a multimodal dialogue system. In Proceedings of the IJCAI Workshop: Knowledge and Reasoning in Practical Dialogue Systems, pages 8-14, Seattle, WA.

Allauzen, C., M. Mohri, M. Riley, and B. Roark. 2004. A generalized construction of speech recognition transducers. In
International Conference on Acoustics, Speech, and Signal Processing, pages 761-764, Montreal.

Allauzen, C., M. Mohri, and B. Roark. 2003. Generalized algorithms for constructing statistical language models. In Proceedings of the Association for Computational Linguistics, pages 40-47, Sapporo.

Allauzen, C., M. Riley, J. Schalkwyk, W. Skut, and M. Mohri. 2007. Openfst: A general and efficient weighted finite-state transducer library. In Proceedings of the Ninth International Conference on Implementation and Application of Automata, (CIAA 2007), Lecture Notes in Computer Science Vol. 4783, pages 11-23. Springer, Berlin, Heidelberg.

Allen, J., D. Byron, M. Dzikovska, G. Ferguson, L. Galescu, and A. Stent. 2001. Towards conversational human-computer interaction. AI Magazine, 22(4):27-38.

André, E. 2002. Natural language in multimedia/multimodal systems. In R. Mitkov, editor, Handbook of Computational Linguistics. Oxford University Press, Oxford, pages 650-669.

Bacchiani, M. and B. Roark. 2003. Unsupervised language model adaptation. In Proceedings of the International Conference on Acoustics, Speech, and Signal Processing, pages 224-227, Hong Kong.

Bangalore, S. 1997. Complexity of Lexical Descriptions and its Relevance to Partial 
Parsing. Ph.D. thesis, University of Pennsylvania, Philadelphia, PA.

Bangalore, S. and M. Johnston. 2000. Tight-coupling of multimodal language processing with speech recognition. In Proceedings of the International Conference on Spoken Language Processing, pages 126-129, Beijing.

Bangalore, S. and M. Johnston. 2004. Balancing data-driven and rule-based approaches in the context of a multimodal conversational system. In Proceedings of the North American Association for Computational Linguistics/ Human Language Technology, pages 33-40, Boston, MA.

Bangalore, S. and A. K. Joshi. 1999. Supertagging: An approach to almost parsing. Computational Linguistics, 25(2):237-265.

Bangalore, S. and G. Riccardi. 2000. Stochastic finite-state models for spoken language machine translation. In Proceedings of the Workshop on Embedded Machine Translation Systems, pages 52-59, Seattle, WA.

Bangalore, S. and G. Riccardi. 2002. Stochastic finite-state models of spoken language machine translation. Machine Translation, 17(3):165-184.

Bellik, Y. 1995. Interface Multimodales: Concepts, Modèles et Architectures. Ph.D. thesis, University of Paris XI (Orsay), France.

Bellik, Y. 1997. Media integration in multimodal interfaces. In Proceedings of the IEEE Workshop on Multimedia Signal Processing, pages 31-36, Princeton, NJ.

Beutnagel, M., A. Conkie, J. Schroeter, Y. Stylianou, and A. Syrdal. 1999. The AT\&T next-generation TTS. In Joint Meeting of ASA; EAA and DAGA, pages 18-24, Berlin.

Boros, M., W. Eckert, F. Gallwitz, G. Gŏrz, G. Hanrieder, and H. Niemann. 1996. Towards understanding spontaneous speech: word accuracy vs. concept accuracy. In Proceedings of the International Conference on Spoken Language Processing, pages 41-44, Philadelphia, PA.

Brison, E. and N. Vigouroux. 1993. Multimodal references: A generic fusion process. Technical report, URIT-URA CNRS, Universit Paul Sabatier, Toulouse.

Carpenter, R. 1992. The Logic of Typed Feature Structures. Cambridge University Press, Cambridge.
Cassell, J. 2001. Embodied conversational agents: Representation and intelligence in user interface. AI Magazine, 22:67-83.

Cheyer, A. and L. Julia. 1998. Multimodal Maps: An Agent-Based Approach. Lecture Notes in Computer Science, 1374:103-113.

Ciaramella, A. 1993. A prototype performance evaluation report. Technical Report WP8000-D3, Project Esprit 2218, SUNDIAL.

Clark, S. and J. Hockenmaier. 2002. Evaluating a wide-coverage CCG parser. In Proceedings of the LREC 2002, Beyond Parseval Workshop, pages 60-66, Las Palmas.

Cohen, P. R. 1991. Integrated interfaces for decision support with simulation. In Proceedings of the Winter Simulation Conference, pages 1066-1072, Phoenix, AZ.

Cohen, P. R. 1992. The role of natural language in a multimodal interface. In Proceedings of the User Interface Software and Technology, pages 143-149, Monterey, CA.

Cohen, P. R., M. Johnston, D. McGee, S. L. Oviatt, J. Clow, and I. Smith. 1998a. The efficiency of multimodal interaction: A case study. In Proceedings of the International Conference on Spoken Language Processing, pages 249-252, Sydney.

Cohen, P. R., M. Johnston, D. McGee, S. L. Oviatt, J. Pittman, I. Smith, L. Chen, and J. Clow. 1998b. Multimodal interaction for distributed interactive simulation. In M. Maybury and W. Wahlster, editors, Readings in Intelligent Interfaces. Morgan Kaufmann Publishers, San Francisco, CA, pages 562-571.

Dowding, J., J. M. Gawron, D. E. Appelt, J. Bear, L. Cherny, R. Moore, and D. B. Moran. 1993. GEMINI: A natural language system for spoken-language understanding. In Proceedings of the Association for Computational Linguistics, pages 54-61, Columbus, $\mathrm{OH}$.

Ehlen, P., M. Johnston, and G. Vasireddy. 2002. Collecting mobile multimodal data for MATCH. In Proceedings of the International Conference on Spoken Language Processing, pages 2557-2560, Denver, CO.

Flickinger, D., A. Copestake, and I. Sag. 2000. HPSG analysis of English. In W. Wahlster, editor, Verbmobil: Foundations of Speech-to-Speech Translation. Springer-Verlag, Berlin, pages 254-263.

Galescu, L., E. K. Ringger, and J. F. Allen. 1998. Rapid language model development for new task domains. In Proceedings of the ELRA First International Conference on 
Language Resources and Evaluation (LREC), pages 807-812, Granada.

Goffin, V., C. Allauzen, E. Bocchieri, D. Hakkani-Tur, A. Ljolje, S. Parthasarathy, M. Rahim, G. Riccardi, and M. Saraclar. 2005. The AT\&T WATSON speech recognizer. In Proceedings of the International Conference on Acoustics, Speech, and Signal Processing, pages 1033-1036, Philadelphia, PA.

Gorin, A. L., G. Riccardi, and J. H. Wright. 1997. How May I Help You? Speech Communication, 23(1-2):113-127.

Gupta, N., G. Tur, D. Tur, S. Bangalore, G. Riccardi, and M. Rahim. 2004. The AT\&T spoken language understanding system. IEEE Transactions on Speech and Audio Processing, 14(1):213-222.

Gustafson, J., L. Bell, J. Beskow, J. Boye, R. Carlson, J. Edlund, B. Granström, D. House, and M. Wirn. 2000. Adapta multimodal conversational dialogue system in an apartment domain. In International Conference on Spoken Language Processing, pages 134-137, Beijing.

Haffner, P., G. Tur, and J. Wright. 2003. Optimizing SVMs for complex call classification. In International Conference on Acoustics, Speech, and Signal Processing, pages 632-635, Hong Kong.

Hauptmann, A. 1989. Speech and gesture for graphic image manipulation. In Proceedings of CHI '89, pages 241-245, Austin, TX.

Jackendoff, R. 2002. Foundations of Language: Brain, Meaning, Grammar, and Evolution Chapter 9. Oxford University Press, New York.

Johnston, M. 1998a. Multimodal language processing. In Proceedings of the International Conference on Spoken Language Processing, pages 893-896, Sydney.

Johnston, M. 1998b. Unification-based multimodal parsing. In Proceedings of the Association for Computational Linguistics, pages 624-630, Montreal.

Johnston, M. 2000. Deixis and conjunction in multimodal systems. In Proceedings of the International Conference on Computational Linguistics (COLING), pages 362-368, Saarbrücken.

Johnston, M., P. Baggia, D. C. Burnett, J. Carter, D. Dahl, G. McCobb, and D. Raggett. 2007. EMMA: Extensible MultiModal Annotation markup language. Technical report, W3C Candidate Recommendation.

Johnston, M. and S. Bangalore. 2000. Finite-state multimodal parsing and understanding. In Proceedings of the International Conference on Computational Linguistics (COLING), pages 369-375, Saarbrücken.

Johnston, M. and S. Bangalore. 2004. Matchkiosk: A multimodal interactive city guide. In Proceedings of the Association of Computational Linguistics (ACL) Poster and Demonstration Session, pages 222-225, Barcelona.

Johnston, M. and S. Bangalore. 2005. Finite-state multimodal integration and understanding. Iournal of Natural Language Enqineering, 11(2):159-187.

Johnston, M., S. Bangalore, A. Stent, G. Vasireddy, and P. Ehlen. 2002a. Multimodal language processing for mobile information access. In Proceedings of the International Conference on Spoken Language Processing, pages 2237-2240, Denver, CO.

Johnston, M., S. Bangalore, G. Vasireddy, A. Stent, P. Ehlen, M. Walker, S. Whittaker, and P. Maloor. 2002b. MATCH: An architecture for multimodal dialog systems. In Proceedings of the Association of Computational Linguistics, pages 376-383, Philadelphia, PA.

Johnston, M., P. R. Cohen, D. McGee, S. L. Oviatt, J. A. Pittman, and I. Smith. 1997. Unification-based multimodal integration. In Proceedings of the Association of Computational Linguistics, pages 281-288, Madrid.

Joshi, A. and P. Hopely. 1997. A parser from antiquity. Journal of Natural Language Engineering, 2(4):6-15.

Kanthak, S. and H. Ney. 2004. FSA: An Efficient and Flexible C++ Toolkit for Finite State Automata Using On-Demand Computation. In Proceedings of the Association for Computational Linguistics Conference, pages 510-517, Barcelona.

Kaplan, R. M. and M. Kay. 1994. Regular models of phonological rule systems. Computational Linguistics, 20(3):331-378.

Kartunnen, L. 1991. Finite-state constraints. In Proceedings of the International Conference on Current Issues in Computational Linguistics, Universiti Sains Malaysia, Penang.

Koons, D. B., C. J. Sparrell, and K. R. Thorisson. 1993. Integrating simultaneous input from speech, gaze, and hand gestures. In M. T. Maybury, editor, Intelligent Multimedia Interfaces. AAAI Press/MIT Press, Cambridge, MA, pages 257-276. 
Koskenniemi, K. K. 1984. Two-level Morphology: A General Computation Model for Word-form Recognition and Production. Ph.D. thesis, University of Helsinki.

Larsson, S., P. Bohlin, J. Bos, and D. Traum. 1999. TrindiKit manual. Technical report, TRINDI Deliverable D2.2, Gothenburg University, Sweden.

Levenshtein, V. I. 1966. Binary codes capable of correcting deletions, insertion and reversals. Soviet Physics Doklady, 10:707-710.

Mohri, M., F. C. N. Pereira, and M. Riley. 1998. A rational design for a weighted finite-state transducer library. Lecture Notes in Computer Science, 1436:144-158.

Neal, J. G. and S. C. Shapiro. 1991. Intelligent multi-media interface technology. In J. W. Sullivan and S. W. Tyler, editors, Intelligent User Interfaces. Addison Wesley, New York, pages $45-68$.

Nederhof, M. J. 1997. Regular approximations of CFLs: A grammatical view. In Proceedings of the International Workshop on Parsing Technology, pages 159-170, Boston, MA.

Nishimoto, T., N. Shida, T. Kobayashi, and K. Shirai. 1995. Improving human interface in drawing tool using speech, mouse, and keyboard. In Proceedings of the 4th IEEE International Workshop on Robot and Human Communication, ROMAN95, pages 107-112, Tokyo.

Noord, G. 1997. FSA utilities: A toolbox to manipulate finite-state automata. Lecture Notes in Computer Science, 1260:87-108.

Och, F. J. and H. Ney. 2003. A systematic comparison of various statistical alignment models. Computational Linouistics, 29(1):19-51.

Oviatt, S., A. DeAngeli, and K. Kuhn. 1997. Integration and synchronization of input modes during multimodal human-computer interaction. In $\mathrm{CHI}$ '97: Proceedings of the SIGCHI Conference on Human Factors in Computing Systems, pages 415-422, New York, NY.

Oviatt, S. L. 1997. Multimodal interactive maps: Designing for human performance. Human-Computer Interaction, 12(1):93-129.

Oviatt, S. L. 1999. Mutual disambiguation of recognition errors in a multimodal architecture. In Proceedings of the Conference on Human Factors in Computing Systems: $\mathrm{CHI}^{\prime} 99$, pages 576-583, Pittsburgh, PA.

Papineni, K. A., S. Roukos, and T. R. Ward. 1997. Feature-based language understanding. In Proceedings of

European Conference on Speech

Communication and Technology, pages 1435-1438, Rhodes.

Pereira, F. C. N. and M. D. Riley. 1997. Speech recognition by composition of weighted finite automata. In E. Roche and Y. Schabes, editors, Finite State Devices for Natural Language Processing. MIT Press, Cambridge, MA, USA, pages $431-456$.

Pieraccini, R. 2004. Spoken language understanding: The research/industry chasm. In HLT-NAACL 2004 Workshop on Spoken Language Understanding for Conversational Systems and Higher Level Linguistic Information for Speech Processing, Boston, MA.

Pollard, C. and I. A. Sag. 1994. Head-Driven Phrase Structure Grammar. Center for the Study of Language and Information, University of Chicago Press, IL.

Punyakanok, V., D. Roth, and W. Yih. 2005. Generalized inference with multiple semantic role labeling systems shared task paper. In Proceedings of the Annual Conference on Computational Natural Language Learning (CoNLL), pages 181-184, Ann Arbor, MI.

Rambow, O., S. Bangalore, T. Butt, A. Nasr, and R. Sproat. 2002. Creating a finite-state parser with application semantics. In Proceedings of the International Conference on Computational Linguistics (COLING 2002), pages 1-5, Taipei.

Ramshaw, L. and M. P. Marcus. 1995. Text chunking using transformation-based learning. In Proceedings of the Third Workshop on Very Large Corpora, pages 82-94, Cambridge, MA.

Riccardi, G., R. Pieraccini, and E. Bocchieri. 1996. Stochastic automata for language modeling. Computer Speech and Lanquage, 10(4):265-293.

Rich, C. and C. Sidner. 1998. COLLAGEN: A collaboration manager for software interface agents. User Modeling and User-Adapted Interaction, 8(3-4):315-350.

Ringger, E. K. and J. F. Allen. 1996. A fertility channel model for post-correction of continuous speech recognition. In International Conference on Spoken Language Processing, pages 897-900, Philadelphia, PA.

Ristad, E. S. and P. N. Yianilos. 1998. Learning string-edit distance. IEEE Transaction on Pattern Analysis and Machine Intelligence, 20(5):522-532. 
Roche, E. 1999. Finite-state transducers: parsing free and frozen sentences. In A. Kornai, editor, Extended Finite-State Models of Language. Cambridge University Press, Cambridge, pages 108-120.

Rubine, D. 1991. Specifying gestures by example. Computer Graphics, 25(4):329-337.

Schapire, R., M. Rochery, M. Rahim, and N. Gupta. 2002. Incorporating prior knowledge in boosting. In Proceedings of the Nineteenth International Conference on Machine Learning, pages 538-545, Sydney.

Schapire, R. E. 1999. A brief introduction to boosting. In Proceedings of International Joint Conference on Artificial Intelligence, pages 1401-1406, Stockholm.

Souvignier, B. and A. Kellner. 1998. Online adaptation for language models in spoken dialogue systems. In International Conference on Spoken Language Processing, pages 2323-2326, Sydney.

Stent, A., J. Dowding, J. Gawron, E. Bratt, and R. Moore. 1999. The CommandTalk spoken dialogue system. In Proceedings of the $27^{\text {th }}$ Annual Meeting of the Association for Computational Linguistics, pages 183-190, College Park, MD.

Thompson, C. A. and R. J. Mooney. 2003. Acquiring word-meaning mappings for natural language interfaces. Journal of Artificial Intelligence Research, 18:1-44.

van Tichelen, L. 2004. Semantic interpretation for speech recognition. Technical Report W3C.

Wahlster, W. 2002. SmartKom: Fusion and fission of speech, gestures, and facial expressions. In Proceedings of the 1st International Workshop on Man-Machine Symbiotic Systems, pages 213-225, Kyoto.

Walker, M., R. Passonneau, and J. Boland. 2001. Quantitative and qualitative evaluation of DARPA Communicator spoken dialogue systems. In Proceedings of the 39rd Annual Meeting of the
Association for Computational Linguistics (ACL/EACL-2001), pages 515-522,

Toulouse.

Walker, M. A., S. Whittaker, A. Stent, P. Maloor, J. D. Moore, M. Johnston, and G. Vasireddy. 2004. Generation and evaluation of user tailored responses in multimodal dialogue. Cognitive Science, 28(5):811-840.

Walker, M. A., S. J. Whittaker, P. Maloor, J. D. Moore, M. Johnston, and G. Vasireddy. 2002. Speech-Plans: Generating evaluative responses in spoken dialogue. In Proceedings of the International Natural Language Generation Conference, pages 73-80, Ramapo, NY.

Wang, Y. and A. Acero. 2003. Combination of CFG and n-gram modeling in semantic grammar learning. In Proceedings of the Eurospeech Conference, pages 2809-2812, Geneva.

Ward, W. 1991. Understanding spontaneous speech: The Phoenix system. In Proceedings of the International Conference on Acoustics, Speech, and Signal Processing, pages 365-367, Washington, DC.

Wauchope, K. 1994. Eucalyptus: Integrating natural language input with a graphical user interface. Technical Report NRL/FR/5510-94-9711, Naval Research Laboratory, Washington, DC.

XTAG. 2001. A lexicalized tree-adjoining grammar for English. Technical report, University of Pennsylvania. Available at www . cis. upenn. edu/ xtag/ gramrelease.html.

Zettlemoyer, L. S. and M. Collins. 2005. Learning to map sentences to logical form: Structured classification with probabilistic categorial grammars. In Proceedings of the Twenty-First Conference on Uncertainty in Artificial Intelligence (UAI-05), pages 658-66, Arlington, VA. 
